[Vicino Oriente XVI (2012), pp. 201-225]

\title{
IL COMPLESSO DI RICEVIMENTO DEL PALAZZO AYYUBIDE A SHAWBAK*
}

\author{
Martina Rugiadi - Sapienza Università di Roma
}

The reception complex in the citadel of Shawbak incorporates an īwān plan that follows the qā'a model and the tripartite composition of the walls. The comparative study of these and of other architectural features (arches, niches, decoration, coverings) allows placing the complex in the Ayyubid architectural tradition. However, it does not propose a conclusive date for its construction: this could have been during the reign of al-Mu'azzam 'Isa (1197-1226) or in the second quarter of the $13^{\text {th }}$ century.

Keywords: Shawbak, Ayyubid architecture, palace, Jordan, $q \bar{a}$ ' $a$.

La cittadella fortificata di Shawbak - Crac Montréal, Mont Réal o Mons Regalis per i crociati - si trova nella Giordania meridionale, a circa 25 chilometri a nord di Petra. Nei venti anni successivi alla sua espugnazione da parte di Salāh al-Dīn (1189), Shawbak, precedentemente nelle mani dei crociati, vide modificare radicalmente la propria conformazione urbanistica ed architettonica, complice anche il terremoto del $1212^{1}$. Il castello crociato, con funzioni principalmente difensive e di controllo del territorio e con limitate pretese palatine, venne adattato alle esigenze dei nuovi signori ayyubidi che vi si insediarono, riconoscendo l'importanza strategica del sito. Tra i loro interventi costruttivi vi furono quelli dedicati al carattere urbano della cittadella e l'impianto di una costruzione palatina adeguata al prestigio e al rango del suo signore.

I resti di alcune costruzioni all'interno della cittadella, infatti, permettono di identificare il modello di complesso palatino comune, con molteplici varianti, a tutte le cittadelle di epoca ayyubide a noi note. Si tratta di edifici composti da svariate unità architettoniche, in cui gli elementi ricorrenti sono una sala di ricevimento (di dimensioni piuttosto contenute e talvolta scoperta) con $\bar{\imath} w \bar{a} n$, un hammām, un portale monumentale, una moschea. Il complesso palatino di Shawbak si trova all'estremità settentrionale della cittadella, quella più lontana dall'unico varco di accesso alla stessa, e consiste, per quanto ne è stato portato in luce, in una sala principale con alcuni locali adiacenti, qui presi in esame e chiamati

* Colgo questa occasione per ringraziare Guido Vannini per avermi dato 1'opportunità di partecipare alla campagna a Shawbak nel 2007 e avermi affidato lo studio del complesso di ricevimento del palazzo ayyubide, confluito in questo testo. Ringrazio anche Michele Nucciotti e gli altri membri del progetto Shawbak (Università degli Studi di Firenze) per la disponibilità sempre dimostrata. A Robin Brown vanno inoltre i miei ringraziamenti affettuosi per le conversazioni stimolanti su Shawbak e Kerak a Cambridge, MA.

Gli ambienti del complesso di ricevimento di Shawbak sono stati portati alla luce e restaurati dal Dipartimento di Archeologia della Giordania, prima delle indagini archeologiche effettuate da R. Brown nel 1986 e di quelle dell'Università di Firenze a partire dal 2006. Per una storia degli studi su Shawbak si veda Brown (1988a, 8-9, tabb. 1, 5) e Vannini ([ed.] 2007, 5-9). Il testo qui presentato si basa sui dati archeologici disponibili al 2009, in una fase iniziale delle indagini dell'Università di Firenze sul periodo post-crociato a Shawbak (Rugiadi 2007).

1 Del ben più disastroso terremoto del 1170, invece, che provocò gravissimi danni alle cittadelle islamiche della Siria (in particolare ad Aleppo, Homs, Hama etc.), non si hanno notizie circa eventuali danni causati a Shawbak, essendo il suo raggio di azione limitato probabilmente all'area siriana. 
complesso di ricevimento, e in un altro gruppo di ambienti tra loro collegati identificati in un hammām. Già i saggi di scavo effettuati da Robin Brown nel 1986 avevano mostrato che la sala di ricevimento rappresentava solo un nucleo di un più vasto complesso palatino all'interno della cittadella fortificata e avevano fornito dati per l'attribuzione al periodo ayyubide ${ }^{2}$.

In questo testo si prenderanno in esame gli elementi costruttivi e decorativi del complesso di ricevimento, nucleo centrale del complesso palatino, per suggerire proposte di datazione sulla base dei confronti architettonici. In quanto esemplificativi del valore che l'élite ayyubide attribuiva a Shawbak, essi sanciscono il passaggio sociale e culturale tra il periodo dell'occupazione crociata e quello della confederazione ayyubide.

\section{DESCRIZIONE (figg. 1-7)}

Del complesso di ricevimento rimangono tre sale di carattere monumentale (UF1-3), consecutive e con orientamento sud est-est ovest (qui semplificato in est-ovest), tre piccoli vani (UF4-6) e tre corridoi adiacenti (UF7-9) ${ }^{3}$; tutti gli ambienti sono conservati per gran parte dell'elevato e sono stati in parte restaurati in tempi recenti.

Gli elementi distintivi della sala principale UF3 (figg. 2-5), che la identificano in quanto sala di ricevimento, sono la pianta a $\bar{w} w \bar{a} n$ secondo il modello della $q \bar{a}{ }^{\prime} a^{4}$ (fig. 1), e la composizione tripartita degli elevati ${ }^{5}$. L'ambiente si attiene ad una composizione simmetrica a due a due sia in pianta sia in elevato.

Si tratta infatti di una sala quadrata sul cui asse nord-sud si aprono, simmetrici, due $\bar{\imath} w \bar{a} n$ poco profondi, entrambi con volta a botte e arco ribassato sulla facciata; quest'ultimo è affiancato da due piccoli archi ribassati ${ }^{6}$ che immettono nei corridoi. Sull'asse est-ovest sono collocati, anch'essi simmetrici, due grandi archi (larghezza 192,5 e 194,5 cm) probabilmente a sesto acuto leggermente ribassato, sorretti da pilastri quadrati, ciascuno affiancato da due piccoli archi ribassati. La triplice apertura del lato est immette in un unico ambiente, posizionato sullo stesso asse est-ovest; sul lato opposto, ad ovest, recenti indagini hanno confermato una composizione uguale e simmetrica (cfr. n. 3).

La sala era molto probabilmente coperta da una volta a crociera ribassata, alta circa 7 $\mathrm{m}^{7}$, o da una volta a crociera con impluvium centrale ${ }^{8}$ (fig. 2).

Brown 1988a, 110; 1988b, 230, 232, tab. 1.

3 L'aspetto più importante della planimetria completa, aggiornata al 2010, sono due sale opposte e simmetriche a UF1 e UF2, sempre sull'asse est-ovest (Vannini - Nucciotti [eds.] 2007, 30; Nucciotti in stampa, fig. 5); i loro rinvenimento, successivo all'elaborazione di questo testo, ne conferma sostanzialmente le considerazioni.

4 La $q \bar{a}$ ' $a$ è la sala di ricevimento, pubblico o privato, che caratterizza l'architettura residenziale del Cairo del periodo mamelucco, ma il termine può essere applicato anche a edifici di periodo precedente (per una discussione sull'uso del termine $\S 2$.1.1.).

5 Cioè con tre aperture (nicchie, $\bar{\imath} w a \bar{n}$, passaggi) su ciascun lato (Tabbaa 1997, 92-93; cfr. § 2.1.3.).

6 Si veda Vannini - Nucciotti ([eds.] 2007, 21, fig. 18) per l'interpretazione della forma originaria di questi archi, compromessa dalla mancanza di tutti i blocchi d'imposta originali e dagli interventi di restauro.

7 Vannini - Nucciotti 2007, 17.

8 Brown (1988a, 50) sostiene invece che si trattasse di una "mitered vault", una volta a crociera con uncini. 
Sulla parete est e tutt'intorno ai pilastri quadrati, all'altezza dell'imposta dei piccoli archi laterali, si trova un alloggiamento rettangolare, destinato ad ospitare forse un architrave in legno (§ 2.2., pp. 210-212; fig. 3).

Un altro alloggiamento rettangolare (altezza 9,5-10 $\mathrm{cm}$ ), volto ad ospitare un inserto decorativo (probabilmente una cornice in legno scolpito e dipinto, $\S 2.4$.), corre in orizzontale lungo tutti i muri settentrionali della sala ad un altezza di circa 1 metro; esso prosegue anche per la larghezza del piccolo arco laterale di nord ovest fino alla linea esterna del piedritto e si inserisce anche nella larghezza dei piccoli archi laterali a nord (figg. 3-4); alcuni profondi fori quadrati praticati nei muri (profondità $44-47 \mathrm{~cm}$, misura del lato $9,5-10 \mathrm{~cm}$ ) erano utilizzati probabilmente per assicurare saldamente l'inserto decorativo rimanendo invisibili.

Le altre due sale sull'asse est-ovest (UF1-2) sono entrambe a pianta rettangolare ma di dimensioni progressivamente minori rispetto alla sala principale, della quale ripropongono alcune forme architettoniche (fig. 1). In particolare vi sono varianti delle composizioni tripartite in cui sono presenti, vero leit-motif di tutto il complesso, piccoli archi ribassati attinenti a passaggi e a nicchie in tutto simili per forma e dimensione (larghezza $98 \mathrm{~cm}$ circa $)^{9}$ a quelli della sala principale.

La sala mediana UF2 (fig. 6) presenta sul suo asse nord-sud rispettivamente una nicchia e un piccolo arco passante, simmetrici. Sul lato est, invece, una soluzione tripartita che richiama quella della sala principale - unica variante è l'arco centrale, che qui è ribassato e di dimensioni minori (larghezza 148,5 cm) - permette il passaggio all'ultima sala. L'ambiente UF2, di altezza minore della sala principale, era probabilmente coperto da una volta a botte, con uncini ${ }^{10}$.

L'ultima sala UF1, la più angusta delle tre, presenta tre nicchie sul lato est - la nicchia centrale più larga delle altre (cfr. n. 9) - e, simmetrici, una nicchia sul lato nord e un passaggio sul lato sud (fig. 1); la sala aveva un'altezza inferiore rispetto anche a quella dell'ambiente mediano ed era coperta da una volta a botte (quella visibile oggi è di restauro). Le tre nicchie del muro est presentano ciascuna un'intercapedine verticale che le separa dal muro retrostante, funzionale probabilmente alla ventilazione dell'ambiente. In tutte e tre, inoltre, sono visibili dei fori rettangolari praticati profondamente nel muro all'altezza dell'imposta dell'arco allo scopo forse di assicurare un architrave ligneo sotto l'arco (§ 2.2., pp. 210-212; fig. 7).

I piccoli archi passanti che si aprono sui lati sud delle tre sale permettono l'accesso ad un corridoio a "elle" originariamente coperto con volta a botte (UF7; fig. 1) e a tre piccoli vani di pianta quadrata coperti con volte a botte (orientamento nord-sud) e connessi tra loro (UF4-6; fig. 1). Ciascuno dei tre vani presenta una nicchia ad arco ribassato sul muro meridionale, ma solo in UF4 la nicchia si trova in asse con il passaggio (in questa nicchia è visibile un profondo foro rettangolare come nelle nicchie in UF1 [fig. 7]; probabilmente anche le nicchie in UF4-6 avevano intercapedini verticali). I piccoli archi passanti che si

$9 \quad$ Le dimensioni dei piccoli archi o nicchie sono (in $\mathrm{cm}$ ): 96; 96,5; 97,4; 97,5; 98 (8 casi); 98,5 (4 casi); 99 ; 103,5. Significativamente più ampia sembra essere la nicchia centrale del muro sud di UF1, larga $109,5 \mathrm{~cm}$.

10 Informazione personale di M. Nucciotti. Brown (1988a, 50) sostiene invece che si trattasse di una volta a crociera. 
aprono sul lato nord della sala principale permettono l'accesso ad un ambiente oggi interrato (UF8; figg. 1, 4), forse un corridoio simmetrico a UF7, e a un lungo corridoio con volta a botte, particolarmente ben preservato (UF9; fig. 1). Tutti questi ambienti minori davano accesso presumibilmente ad altre aree del palazzo (cfr. n. 112).

Ad ovest della sala principale si trovavano due sale uguali e simmetriche a UF1 e UF2 (cfr. n. 3).

Le tre sale principali e le soglie, gli stipiti e gli archi dei piccoli passaggi e delle nicchie degli ambienti minori sono in conci di calcare micritico, molto ben lavorati ${ }^{11}$; gli elevati degli ambienti minori sono in blocchi sbozzati, sempre di calcare micritico ${ }^{12}$; quanto alle coperture, quelle a botte sono di pietre sbozzate di diversi litotipi.

Gli elementi architettonici del complesso di ricevimento sono impostati così da enfatizzare la preminenza della sala principale: le dimensioni gradualmente maggiori degli ambienti, sia in pianta sia in elevato, fino alla massima ampiezza e altezza della sala principale; la sua collocazione centrale (considerando lo sviluppo del complesso verso ovest), allo sbocco di tutti i corridoi che portavano nelle diverse aree del palazzo; la presenza dei due $\bar{w} w \bar{a} n$ contrapposti e lo sviluppo simmetrico degli elementi architettonici che la compongono (è l'unica sala a pianta quadrata del complesso); la fascia decorativa. Tra le caratteristiche architettoniche che restituiscono l'idea di una progettazione organica e pianificata del complesso, mirata a concentrare l'attenzione sulla sala principale, è anche il livello pavimentale di quest'ultima, il cui spazio centrale, o durq $\bar{a}$ ' $a{ }^{13}$, è inferiore non solo rispetto agli $\bar{w} w \bar{a} n$ ma soprattutto alla sala adiacente, a sua volta di poco più bassa dell'ultima sala a est ${ }^{14}$.

La fascia decorativa, che correva solo sui muri nord, interrompe la simmetria della struttura, stabilendo così il primato di questa metà dell'ambiente sull'altra; questo primato è confermato dalla presenza delle nicchie sul solo lato settentrionale delle altre due sale, nicchie sulle quali, piuttosto che sui passaggi verso gli ambienti minori del lato meridionale, si focalizza l'attenzione del visitatore. È quindi probabile che durante le sessioni di ricevimento il governatore si collocasse nell' $\bar{w} w \bar{a} n$ nord.

\section{GLI ELEMENTI ARCHITETTONICI}

\subsection{La qā'a con cortina tripartita}

\subsubsection{La $q \bar{a} ' a$}

Il modello a $\bar{l} w a \bar{n}$ riconoscibile nell'ambiente principale caratterizza, con molteplici varianti, tutti i palazzi di epoca ayyubide a noi noti, nonché molti dei complessi residenziali o di ricevimento identificati nelle fortificazioni e negli edifici religiosi (madrasa, mašhad,

11 Le finiture dei conci evidenziati sono di tipo S6, S9, S6.1; per gli elevati degli esterni di tipo S23 (Vannini Nucciotti [eds.] 2007, 19; cfr. n. 110).

12 Vannini - Nucciotti 2007, 20.

13 Il termine viene generalmente utilizzato per gli edifici residenziali del Cairo, mentre in Siria è talvolta sostituito da 'ataba (David 2007, 62).

14 Vannini - Nucciotti (eds.) 2007, 19. 
hanqāh) dello stesso periodo ${ }^{15}$. Si tratta di una pianta che prevede due, tre o quattro $\bar{\imath} w \bar{a} n$ (assumendo in quest'ultimo caso un aspetto cruciforme), spesso rialzati ${ }^{16}$, affacciati su un ambiente centrale dalle dimensioni piuttosto ridotte. Tra i monumenti di XII e XIII secolo con funzione residenziale che presentano questa pianta citiamo (per quanto possibile in ordine cronologico): Qașr al-Banāt a Raqqa ${ }^{17}$, Qal' at Șahyūn ${ }^{18}$, il palazzo nella cittadella di Aleppo ${ }^{19}$, il palazzo di Diyarbakr ${ }^{20}$, il Maṭbah al-'Ağamīi ${ }^{21}, \mathrm{Qal}^{\text {'at }} \mathrm{Nağm}^{22}$, la torre di Aybak nel castello di 'Ajlun ${ }^{23}$, il donjon di Bosra ${ }^{24}$, due unità residenziali nella madrasa alMustanșirīyya a Baghdad ${ }^{25}$, il palazzo di Rawdia ${ }^{26}$ al Cairo e, ad Aleppo, l'ambiente cupolato residenziale nel Mašhad al-Husayn ${ }^{27}$, le due unità residenziali nella madrasa alFirdaws $^{28}$, quella nel hānqqa al-Farāfra ${ }^{29}$, quella cupolata nella madrasa al-'Adīmiyya ${ }^{30}$;

15 Anche i $d \bar{a} r$ al- 'adl ("palazzi di giustizia") e altri edifici con funzioni ancora diverse avevano verosimilmente questa stessa pianta (Rabbat 1995b, 8-9).

16 Il rialzamento del livello degli īwān rispetto all'ambiente centrale ribassato, caratteristica della $q \bar{a}$ ' $a$ mamelucca, è presente a Shawbak così come in altri edifici ayyubidi, tra cui il donjon a Bosra (Abel 1956, 131).

17 Gli studi più recenti attribuiscono questo edificio al 1170 (Toueir 1985, 297-319; vedi anche Hillenbrand 1985, 36-38; Tabbaa 1997, 89, figg. 54-55; Korn 2004, II, 283).

18 Il palazzo è datato a circa il 1188 o, più probabilmente, al 1200-1215 (Tabbaa 1997, 89-90, figg. 56-57; Beddek 2001, 75, 79-80, figg. 4-9; Michaudel 2001, 201-206). Per confronti stilistici Korn (2004, II, 260-262) preferisce attribuirlo agli anni 1232-1242.

19 Del 1193, con interventi successivi (Tabbaa 1997, 84, figg. 31-32, 43-44; Allen 1999, cap. 5; Korn 2004, II, 221-222, fig. 43; Gonnella 2006, 170; 2007, figg. 93, 95, 106). All'interno della cittadella si trovano i resti di un altro edificio palatino, meno noto, detto palazzo Țawāši e risalente probabilmente al 1230-31: anch'esso ha una pianta a $\bar{\imath} w \bar{a} n$ con composizione tripartita delle facciate (Gonnella 2007, fig. 93; Korn 2004, II, 237).

20 Degli inizi del XIII secolo (Aslanapa 1962, 118-120, 126-127; Hillenbrand 1994, 417; Tabbaa 1997, 88, 91, fig. 61).

21 Del XIII secolo (Tabbaa 1997, 90-91, fig. 62); Korn (2004, II, 240, 253-254, fig. 52) ipotizza che possa trattarsi del lascito testamentario di Šams al-Dīn Abū Bakr Aḥmad Ibn al-'Ağamī della sua residenza privata quale hāanqā; in tal caso sarebbe datato al 1233-1234.

22 Costruito intorno al 1210 (Sourdel 1974, 482; Tabbaa 1997, 90 nota 59, figg. 58-60, 68; Korn 2004, II, 240; Tabbaa 2006, figg. 8b, 10).

23 Costruita nel 1214 (Korn 2004, II, 96, fig. 13; Yovitchitch 2006, 234, figg. 4, 6-7).

24 Del 1213-14 (Abel 1956, 107-108, tav. VII; Tabbaa 1997, fig. 65; Korn 2004, II, 176, fig. 32).

25 Del 1227-1234 (Creswell 1959, II, 126, fig. 68). Creswell le definisce $q \bar{a}$ ' $a$.

26 Costruito tra il 1240 e il 1243 (Creswell 1959, II, 84-87, fig. 38; Hillenbrand 1994, 435; Rabbat 1995a, 87; Pringle 1997, 66; Garcin 2001, 77; Korn 2004, II, 35, fig. 7).

27 L'ambiente residenziale è attribuito alla fase costruttiva del Mašhad databile tra il 1216 e il 1237 (Tabbaa 1997, 120-121, figg. 88, 107); Korn (2004, II, 218-219, 237-238, fig. 41) lo attribuisce al periodo tra il $1231 \mathrm{e}$ il 1236.

28 Del 1237 (Herzfeld 1954-1956, 297-302, tavv. 128-33; Tabbaa 1997, 159, figg. 178-180; Allen 1999, cap. 8; Korn (2004, II, 242-244, fig. 49) la attribuisce al periodo tra il 1235-1236 e il 1241.

29 Si tratta del secondo ambiente, coperto da una volta a crociera con grande impluvium; l'edificio è del 1238 (Tabbaa 1997, 167, fig. 184; Korn 2004, II, 245-246, fig. 50).

30 Ambiente nord est della madrasa, attribuito alla fase costruttiva tra il 1241 e il 1251; particolarmente simile al donjon ayyubide di Bosra (Tabbaa 1997, 137, 159-160 fig. 117a). Korn 2004, II, 247-248, fig. 51 (madrasa Kamālīyya 'Adīmiyya). 
probabilmente utilizzano un impianto simile anche l'edificio palatino a Harim $^{31}$ e quello a Qa'lat Ğind nel Sinai ${ }^{32}$; infine, la sala di ricevimento di Karak ${ }^{33}$.

Tale modello di sala di ricevimento a $\bar{\imath} w \bar{a} n$, i cui antecedenti possono ricercarsi anche nelle abitazioni con corte e $\bar{\imath} w \bar{a} n$ portate alla luce durante gli scavi di Fustat e attribuite ai periodi tulunide e fatimide, ha un corrispettivo vernacolare nelle abitazioni di XII e XIII secolo rinvenute a Mayadin, Balis-Meskene e Rusafa ${ }^{34}$. Tali edifici, che si sviluppano gli uni accanto agli altri in maniera spontanea e in cui manca l'adesione ad un progetto simmetrico, suggeriscono l'esistenza di una tradizione locale che ha sicuramente concorso alla formazione della pianta simmetrica a $\bar{\imath} w \bar{a} n$ degli edifici palatini ayyubidi ${ }^{35}$.

In epoca mamelucca l'ambiente di ricevimento degli edifici si standardizza nel modello a due $\bar{i} w \bar{a} n$ - con copertura piatta - contrapposti e affacciati su uno spazio centrale coperto (detto durqā'a), cui ci si riferisce con il termine $q \bar{a}^{\prime} a$. Tale denominazione, solitamente riservata agli edifici mamelucchi, può essere legittimamente adottata anche per quelli anteriori, come si riscontra nelle fonti dove il termine è usato anche in riferimento ad edifici precedenti. Si pensi alla Qā'a al-Șālihịyya costruita al Cairo dal sultano ayyubide al-Malik al-Ṣālih (1205-1249) ${ }^{36}$, o, nella cittadella della stessa città, alla Qā‘a al-‘Awāmīd, voluta probabilmente da Šağarat al-Durr, sultana nel 1250, come luogo di ricevimento ufficiale (vi

31 L'ipotesi è dedotta dalla pianta, sebbene questa venga pubblicata in dimensioni molto ridotte e forse in forma non definitiva, e dalla descrizione che ne segue: il palazzo, che si sviluppava su più livelli per la presenza di una scala, era diviso in due sezioni, quella meridionale con lunghi ambienti rettangolari (deposito munizioni, magazzini), quella superiore con «a central square room [...] (a kind of reception hall?) which [sic] three areas leading off it»; nei pressi dell'area di ricevimento era anche il hammām del palazzo. L'edificio è attribuito al periodo tra la fine del XII e gli inizi del XIII secolo (Gelichi 2006, 194, fig. 2). Nella pianta pubblicata in Korn (2004, II, 268-270, fig. 57) due $\bar{\imath} w \bar{a} n$ si aprono sulla corte.

32 Feïss-Jehel et al. (2008), fig. 0. Hillenbrand (1994, 415) menziona anche Mardin. Anche per la cittadella di epoca ayyubide a Raqqa, che era probabilmente strutturata intorno ad una corte centrale, non è da escludere un impianto a quattro $\bar{w} w \bar{a} n$ (Heidemann 2006, 136; il sito archeologico è oggi inglobato nella città moderna). La pianta ad $\bar{\imath} w \bar{a} n$, rialzati, si ritrova anche in alcuni ambienti delle torri di cinte murarie fortificate, ad esempio al Cairo: nella torre quadrata nord ovest della cittadella (con ambiente centrale cupolato) e nel Burğ Șuffa, Burğ al-Ṭurfa, Burğ Kerkyalān (con ambiente centrale con volta a crociera), tutti datati all'epoca di al-'Ādil, 12001218 (Creswell 1959, II, figg. 2-3, 5, 13).

33 La datazione di questo edificio al periodo ayyubide o mamelucco è controversa. R. Brown (1989, 290), che vi ha effettuato un'indagine archeologica, lo attribuisce al 1311 sulla base dell'assegnazione al periodo mamelucco dei reperti provenienti dallo scavo. Tale datazione non è unanimemente accettata da altri, che preferiscono il periodo ayyubide tra il 1227 e il 1229 (Pringle 1997, 59; Tabbaa 1997, 88; vedi anche Milwright 2008, 86, n. 169, che pure preferisce aderire all'attribuzione al periodo mamelucco di R. Brown). L'impianto architettonico presenta una similarità quasi pedissequa con il complesso di ricevimento di Shawbak (§ 2.1.3., pp. 208-209; § 2.2., pp. 210-212), mentre la tecnica costruttiva utilizzata - pietre appena sbozzate - non mostra la stessa competenza nelle finiture; si può ipotizzare che la sua realizzazione, che sia avvenuta nel periodo ayyubide o in quello mamelucco, sia stata comunque ispirata a quella di Shawbak e quindi posteriore.

34 Rousset 1996, 187-188, figg. 2-5 (per Mayadin); David 2007, figg. 45-46, 56, e n. 8. Lo studioso suggerisce che lo stesso modello si ritrovi anche a Harran e a Ba'albek ma non fornisce riferimenti.

35 David 2007, 56. Hillenbrand $(1985,37)$ lo ritiene invece un elemento di importazione orientale (Iraq o Iran); Korn (2008, 107-108) sostiene che la pianta a quattro ìwān sia stata importata dall'Iran, pur menzionando l'esistenza delle tradizioni locali attestate nei siti sulla riva occidentale dell'Eufrate.

36 Distrutto in un incendio nel 1284-1285; secondo al-Zāhịīi (XV secolo) aveva le funzioni precedentemente espletate dal qașr (Behrens-Abouseif 1988, 35). 
si teneva una quotidiana processione musicale ${ }^{37}$; risalendo nel tempo rintracciamo l'uso del termine $q \bar{a}^{\prime} a$ con un significato simile in un documento già alla fine del XII secolo ${ }^{38}$, senza contare che alcuni ambienti dei palazzi fatimidi erano denominati con il termine $q \bar{a}{ }^{\prime} a^{39}$. Tra i monumenti preservati, l'esempio più antico di $q \bar{a}^{\prime} a$ al Cairo, che risale probabilmente alla metà del XII secolo, è la Q $\bar{a}^{`} a$ al-Dardīir ${ }^{40}$, in cui si può già evidenziare un'evoluzione verso la pianta della $q \bar{a}^{\prime} a$ con due $\bar{i} w \bar{a} n$ tipica del periodo mamelucco. Il termine $q \bar{a}^{\prime} a$ dunque può essere utilizzato anche per edifici pre-mamelucchi come Shawbak ${ }^{41}$.

Le specificità della $q \bar{a}^{\prime} a$ a Shawbak includono la compresenza di elementi non riscontrabili in tutti gli edifici coevi menzionati, in particolare la presenza di piccoli $\bar{\imath} w \bar{a} n$, la tripartizione degli elevati, la copertura dell'ambiente centrale (§ 2.5.).

\subsection{2. $\bar{I} w \bar{a} n / \mathrm{suffa}$}

I due ambienti contrapposti che abbiamo chiamato ìwān possono essere meglio identificati con il termine șuffa ${ }^{42}$, che può indicare infatti un $\bar{i} w \bar{a} n$ di dimensioni ridotte, non una vera e propria sala ma una sorta di recesso o grande nicchia, proprio come quelli di Shawbak (larghezza circa $2,8 \mathrm{~m}$, profondità $2,1 \mathrm{~m})^{43}$; a Shawbak sono entrambi rialzati. Piccoli $\bar{\imath} w \bar{a} n$ o șuffa erano impiegate in Egitto, insieme all' $\bar{w} w \bar{a} n$ vero e proprio, già in epoca fatimide $^{44}$. I monumenti che conservano elementi di questo tipo sono almeno otto: Șahyūn (3 șffa rialzate), palazzo della cittadella di Aleppo (2 suffa rialzate nella corte piccola), Diyarbakr (1 șuffa), Maṭbah al-'Ağamī (1 șuffa rialzata), Qal'at Nağm (2 șuffa rialzate), Mašhad al-Husayn (1 suffa), madrasa al-Mustanșirīyya (2 șuffa aperte sulla corte centrale), madrasa al-Firdaws (1 șuffa rialzata sia nell'unità residenziale nord est sia in quella nord ovest).

\subsubsection{Cortina tripartita/mağlis}

La composizione tripartita delle facciate rientra nelle caratteristiche della maggior parte degli ambienti palatini di epoca ayyubide, tutti siriani, sebbene solo il Qașr al-Banāt a Raqqa e Karak presentino questa composizione in tutte e quattro le facciate come a

37 Sotto i mamelucchi Bahri, quando ogni moglie o concubina del sultano abitava in una propria $q \bar{a}$ ' $a$, divenne la residenza della moglie favorita del sultano (Behrens-Abouseif 1988, 52-54).

38 Il termine è impiegato in relazione ad un edificio descritto in uno dei documenti della Geniza, in lingua araba ma in caratteri ebraici (Goiten 1977, 163-178).

39 Famosa, ad esempio, è la Qā'a al-Dahab ("dell'oro") che si trovava nel palazzo fatimide costruito dal califfo al-'Azīz (975-996) e rinnovato da al-Mustanșir (1036-1094), di cui abbiamo le descrizioni di al-Maqrīzī (1364-1442) e al-Qalqašandī (m. 1418). Sayyid 1987, 37

40 Creswell 1959, I, 261-263, fig. 159; Lézine 1972a, 120-123, fig. 23.

41 Aggiungiamo che Creswell $(1959,208)$ e Lézine (1972a) chiamano qā'a il palazzo di Rawḍa; David (2007, nota 12) ritiene opportuno utilizzare il termine per gli ambienti di ricevimento a Saḥyūn.

42 Il termine è utilizzato per descrivere una serie di elementi architettonici (cfr. Bloom 1987, 7, n. 6 per i significati di "porticato", "īwān", "recesso").

43 Già Brown (1988b, 227) si era mostrata restia a chiamare $\bar{w} w \bar{a} n$ i recessi di Shawbak.

44 Sayyid 1987, 51, e n. 1. Interessante l'impiego di vere e proprie nicchie di pochi decimetri di profondità: in Egitto si ritrova già nel palazzo di Rawḍa (1240-1243) e nella Qā‘a al-Dardīr (XII secolo; tuttavia Lézine 1972a, fig. 23, 120-123, 130 sembra preferire una datazione al XIV secolo); sono inoltre comuni nei complessi con $q \bar{a}$ ' $a$ di XIV secolo (Lézine 1972a). 
Shawbak ${ }^{45}$. A Shawbak la tripartizione prevede passaggi che introducono in corridoi diversi sui lati degli ìwān/ṣuffa (tutti i lati con șuffa dei monumenti summenzionati sono tripartiti), mentre nei lati rimanenti le tre aperture - delimitate da due pilastri che sorreggono l'arco di quella centrale - introducono in un unico ambiente retrostante. Quest'ultima disposizione l'apertura tripartita verso un unico ambiente rettangolare, o cortina tripartita, chiamata da alcuni studiosi "screen wall"46, "tripartite entry" 47 o anche "travée rythmique" 48 - è anch'essa frequente nei monumenti siriani. Secondo l'interpretazione di Sayyid ${ }^{49}$ si tratta dell'elemento indicato nella letteratura araba con il termine mağlis; quest'ultimo potrebbe dunque essere il termine più appropriato.

Delle dieci unità residenziali siriane note che presentano la tripartizioni degli elevati ${ }^{50}$ quelle con cortina tripartita/mağlis sono sette: il palazzo nella cittadella di Aleppo (entrambe le corti hanno 3 lati tripartiti, 1 mağlis nella corte piccola sembra non rialzato), il Mațbah al-'Ağamī (3 lati tripartiti, 2 mağlis), il Qal'at Nağm (3 lati tripartiti, 1 mağlis rialzato), il solo lato preservato nella torre di Aybak nel castello di 'Ajlun' (1 lato tripartito, 1 mağlis preservato), Karak (2 lati tripartiti, 2 mağlis), il donjon a Bosra (2 lati tripartiti, 2 mağlis), le due unità residenziali nella madrasa al-Firdaws (1 lato tripartito, 1 mağlis in entrambe).

Il modulo cortina tripartita/mağlis a Shawbak è riproposto simmetricamente sulle due facciate opposte dell'asse est-ovest, come avviene unicamente nel Mațbah al-'A ğamī ad Aleppo e nel donjon a Bosra; solo a Shawbak, tuttavia, la pianta è resa più complessa dalla riproposizione della cortina tripartita altre volte ancora sull'asse est-ovest, tra gli ambienti minori (UF2 e UF1; fig. 6) ${ }^{52}$. Un antecedente di tale disposizione si riscontra solo nell'architettura religiosa, nella madrasa di Gümüštekin a Bosra, del 1136 (la più antica madrasa conservatasi in Siria) ${ }^{53}$; in Egitto si veda la moschea di Lu'lu'a al Cairo $(406 / 1015-16)^{54}$

45 Tabbaa $(1997,92-93)$ ne rintraccia la tradizione a partire dai Parti, forse ispirati dagli archi trionfali dei Romani; Parti e Sasanidi usarono la "facciata tripartita" in combinazione con l'̄wwān, come ritroviamo in epoca islamica a Kufa, Mshatta, 'Ukhaidir, Samarra, Lashkari Bazar. Tabbaa (1997, 92-93, con bibliografia) propone che la fusione tra l'ìwān tripartito e la tradizione architettonica locale siriana abbia generato la forma impiegata nell'architettura ayyubide (un prototipo è da rintracciare nelle chiese del nord della Siria)

46 Allen 1999.

47 Tabbaa 1997, 90

48 Yovitchitch 2006, 237; il termine, tuttavia, è forse utilizzato nel senso generico di tripartizione.

49 Sayyid 1987, 35-36.

50 Gli edifici con composizioni tripartite ma senza maǧlis sono: Qașr al-Banāt a Raqqa (4 lati tripartiti), Qal'at Șahyūn (3 lati tripartiti), l'ambiente cupolato residenziale nel Mašhad al-Ḥusayn ad Aleppo (1 lato tripartito). Tabbaa 1997, 120-121, figg. 88, 107.

51 Yovitchitch 2006, 236-237, fig. 7.

52 Nell'architettura religiosa questo modulo spesso è proprio di un ambiente rettangolare suddiviso in tre zone delle quali la centrale cupolata. Ad esempio ad Aleppo: madrasa al-Šādbahtiyya (1193), madrasa alKāmilīyya (1230-37), madrasa al-Șulțāniyya (1233), ḩānqā al-Farāfra (1238), madrasa al-Šarafiyya (terminata nel 1242), madrasa al-“Adīmiyya (1241-1251). Tabbaa 1997, figg. 112, 114, 116, 117a, 119, 184

53 Datata 1136; da notare che gli īwān hanno una copertura piatta (Tabbaa 1997, fig. 109; Allen 1999, figg. 1617).

54 Creswell 1959, I, 113-115, fig. 53, tav. 35 
In definitiva gli elementi distintivi dell'impianto a $\bar{\imath} w \bar{a} n$ e della composizione tripartita del complesso residenziale di Shawbak ben si inscrivono nell'architettura residenziale ayyubide. I confronti più immediati sono il donjon a Bosra del 1213-14 (2 mağlis, volta a crociera con impluvium), la corte piccola del palazzo della cittadella di Aleppo e il Qal'at Nağm (entrambi con 2 suffa simmetriche, rispettivamente del 1193 e del 1210 c.). Forse significativamente, nessun altro palazzo presenta ripetuto il modulo del mağlis. La qa' 'a del complesso palatino di Karak ha una composizione quasi identica a quella di Shawbak unica variante è l'utilizzo invertito del tipo di archi, a sesto acuto per gli $\bar{\imath} w \bar{a} n$ e a sesto ribassato per i passaggi -, ma se ne distingue visibilmente per le finiture molto grossolane utilizzate nella costruzione ${ }^{55}$.

\subsection{Gli archi}

Le osservazioni che seguono, benché non esaustive, si avvalgono dei dati emersi dall'analisi stratigrafica muraria ${ }^{56}$ e dal raffronto con altri esempi nell'architettura dei secoli XII e XIII, per proporre ipotesi interpretative dei metodi di costruzione e della forma originaria degli archi presenti a Shawbak. Tutti gli archi oggi visibili nel complesso di ricevimento - a sesto acuto (forse ribassato) i grandi archi sorretti dai pilastri della sala principale, a tutto sesto ribassato le nicchie e i piccoli passaggi e l'arco centrale tra UF2 e UF3 (fig. 3) - sono stati sottoposti a restauri che ne hanno in parte modificato la forma originaria; in particolare nessuna delle imposte sembra essere autentica ${ }^{57}$. Unica e notevole eccezione sono gli archi a tutto sesto ribassato degli $\bar{\imath} w \bar{a} n$, che conservano anche le imposte originarie di forma triangolare.

La struttura degli $\bar{\imath} w \bar{a} n$ di Shawbak è straordinaria nel panorama del XII e XIII secolo, non trovando riscontro né negli īwān degli edifici o strutture residenziali del XII e della prima metà del XIII secolo preservatisi fino alla parte superiore degli $\operatorname{archi}^{58}$, né in nessun altro edificio 59

Tuttavia, sebbene gli archi a sesto ribassato non siano stati frequentemente impiegati per gli $\bar{w} w \bar{a} n$ o quali grandi archi di passaggio nell'architettura ayyubide, molte sono le varianti simili, tra cui un unico caso a noi noto di arco ribassato poggiante su un architrave ligneo

55 Brown 1989, figg. 2-3.

56 Vannini - Nucciotti (eds.) 2007, 17-22; Nucciotti 2007, 44-45.

57 Vannini - Nucciotti (eds.) 2007, 21. Non siamo a conoscenza di alcuna documentazione fotografica precedente ai restauri.

58 Solo sei edifici residenziali sono così ben preservati negli elevati: Qașr al-Banāt (i resti dell'arco hanno permesso l'identificazione della forma originaria, Toueir 1985, 309, figg. 4-5, tavv. 75:a-d; Tabbaa 1997, fig. 55), Qal'at Nağm (Tabbaa 1997, 90, figg. 59, 68), Mațbah al-'Ağamī (Tabbaa 1997, figg. 63-64), donjon a Bosra (Abel 1956, tav. VII), Karak (Brown 1989, 290).

59 Notiamo la presenza di un arco a tutto sesto ribassato con imposte triangolari a Burzay (Hișn Burzay o Qal'a Mirza), nella valle dell'Oronte: visibile sulla facciata esterna della torre 3, avrebbe dovuto costituire l'ingresso principale alla fortificazione. Nonostante l'assenza di dati cronologici assoluti è stato ipoteticamente attribuito al periodo crociato (Dangles et al. 2001, 24, fig. 8, pianta 7). Alcuni archi apparentemente a sesto ribassato oggi visibili sugli edifici sono invece dovuti a restauri di epoca posteriore: ad esempio nel portale di Ibn Turayra del Mašhad al-Muhassin (594/1197-98) nei pressi di Aleppo (Allen 1999, cap. 4, fig. 28; restauro del secolo XIX). 
nella madrasa al-Firdaws ${ }^{60}$, i rari archi a timpano ribassati ${ }^{61}$, o i diversi tipi di archi a sesto ribassato con il profilo superiore dell'estradosso orizzontale, poggianti sempre su un architrave - elemento sul quale torneremo - e usati spesso anche per finestre e piccole aperture; questi ultimi sono molto comuni nel XII e XIII secolo e sono ritenuti da Terry Allen di tradizione damascena ${ }^{62}$. Particolarmente significativo è il confronto con alcuni archi ribassati presenti ad 'Ajlun, sebbene impiegati secondo un modalità costruttiva che prevede l'addossamento di due archi diversi sui due lati di una stessa apertura: sono presenti sia in alcuni ambienti interni della fortificazione ${ }^{63}$, sia nella sala di ricevimento o $q \bar{a}$ ' $a$ della torre di 'Aybak (611/1214-15) - dove il lato che si affaccia sulla durqā' $a$, pur essendo a tutto sesto ribassato, presenta la parte superiore piatta ed è sostenuto da un architrave in pietra ${ }^{64}$. L'interesse di questa composizione risiede nel fatto che sembra unire la tradizione damascena degli archi a sesto ribassato e profilo superiore dell'estradosso orizzontale, poggianti su un architrave, con l'impiego di un arco a tutto sesto ribassato integrale il cui unico altro esempio si trova a Shawbak, suggerendo che quest'ultimo possa rispecchiare una tradizione locale. Gli archi di passaggio (ma non degli $\bar{\imath} w \bar{a} n$ ) della sala di ricevimento a Karak, probabilmente posteriore a Shawbak e ispirata alla sua architettura (cfr. nota 33), dimostrano senz'altro la diffusione degli archi ribassati in questo territorio ${ }^{65}$.

Il problema di individuare il contesto architettonico in cui collocare gli archi a sesto ribassato di Shawbak non riguarda solo gli $\bar{\imath} w \bar{a} n$, bensì anche tutti i piccoli passaggi e le nicchie, e l'arco centrale tra UF2 e UF3 (fig. 3); questi elementi, tuttavia, potrebbero essere stati realizzati in maniera differente. Gli alloggiamenti rettangolari visibili tutt'intorno ai grandi pilastri della sala principale, infatti, all'altezza dell'imposta dei passaggi laterali, molto probabilmente hanno ospitato architravi lignei, secondo un procedimento costruttivo impiegato in alcuni edifici di epoca ayyubide che ancora conservano le travi originarie. Gli esempi preservati si trovano a Damasco, nel Dār al-Hadīth di Nūr al-Dīn ${ }^{66}$ e nella madrasa

60 Nell'unità residenziale a nord est (Tabbaa 1997, fig. 178; cfr. p. 211).

61 Ad esempio gli archi maggiori sull'asse est-ovest del Maṭbah al- 'Ağamī ad Aleppo (Tabbaa 1997, 91, fig. 63).

62 Esempi: porte a ovest della grande moschea (1109-10) e nella perduta Qubba al-Tawāwis (1103-04) a Damasco; porta della cittadella di Palmira (1132-33); nella Turba al-Nağmiyya (costruita tra il 1146 e il 1166), portale e finestre della madrasa di Nūr al-Dīn (fondata 1167-68), interno dell'ingresso occidentale della Turba Sitt al-Šām al-Suğrā (1173), in cui l'architrave è composto da tre travi lignee, e madrasa al'Azīziyya (1195) a Damasco; il Maqām Ibrāḥīm inferiore (1167-68) ad Aleppo; aperture sulla corte nella madrasa Abu'l-Fawāris a Ma'arrat al-Nu'mān (1199). Varianti più tarde: finestre nel Ğāmi' al-Hanābila (1201-02) e portale est della cittadella (1213-14) a Damasco, hāanqā al-Farāfra ad Aleppo (1238), finestre a est nella madrasa al-Ruqnīyya extra muros (circa 1227), finestre nella madrasa al-Atabakiyya (post 1185), nella madrasa al-Sāhibah (1233), nella Turba Amat al-Latīf (probabilmente 1245-46), nella Turba al-Qaymarī (ante 1256) a Damasco. Allen 1999, cap. 1, figg. 9-12, 15, 20; cap. 2 figg. 29-31, 33; cap. 3 figg. 6, 27; cap. 4 figg. 43-44; cap. 5, figg. 11, 17; cap. 9, figg. 22, 41-42.

63 Molti però sembrano essere leggermente acuti.

64 Solo i passaggi laterali della cortina tripartita si sono preservati completamente; dell'arco centrale rimangono unicamente l'architrave in pietra e il concio d'imposta sul lato della durqā' $a$, da cui si evince che aveva anch'esso uno sviluppo ribassato. Vedi Yovitchitch 2006, fig. 7 (lato sulla durqa' 'a).

65 Brown 1989, 290

66 Edificato tra il 1154 e il 1174 (Sauvaget - Écochard - Sourdel-Thomine 1938-1950, 18, fig. 8; Allen 1999, cap. 2). 
'Ādilīyya ${ }^{67}$, e ad Aleppo, nel Maqām Ibrāḥ̄im inferiore ${ }^{68}$, nella madrasa al-Ẓāhiriyya ${ }^{69}$, nel hăanqā al-Farāfra ${ }^{70}$, nelle due unità residenziali e nella corte della madrasa al-Firdaws ${ }^{71}$, e nel Mațbah al-'Ağamīㄱ.

I due edifici aleppini della madrasa al-Firdaws e del ḩānqā al-Farāfra, per i quali peraltro si ipotizza la mano di un solo architetto ${ }^{73}$, offrono i confronti più significativi per Shawbak. In particolare, nella piccola unità residenziale di nord est nella madrasa alFirdaws, di cui abbiamo già ricordato la pianta del tipo $q \bar{a}^{\prime} a$ con tripartizione degli elevati, le travi lignee sostengono archi ribassati come a Shawbak ${ }^{74}$. Nel hāanqā al-Farāfra, invece, travi lignee corrono anche tutt'intorno ai pilastri della cortina tripartita, oltre che a fungere da architrave per i passaggi laterali, nello stesso modo in cui avrebbero potuto essere collocate a Shawbak, a giudicare dagli alloggiamenti rettangolari predisposti intorno ai pilastri. Nel ḩānqa al-Farāfra, inoltre, il passaggio centrale della cortina tripartita che immette nella sala di preghiera, un arco a sesto acuto, presenta un architrave ligneo su cui poggia l'imposta; anche questa tecnica potrebbe essere stata adottata a Shawbak, in particolare per l'arco di passaggio tra UF2 e UF1 (fig. 6), anch'esso a sesto acuto. Non possiamo infine escludere l'impiego di travi lignee in tutti gli altri archi presenti a Shawbak - i piccoli passaggi, le nicchie, l'arco centrale tra UF2 e UF3 (fig. 3) -, dove le imposte originarie sono perdute; a giudicare dalla conformazione attuale dei blocchi d'imposta, inoltre, gli architravi lignei potrebbero essere stati sormontati da un secondo architrave più spesso, ligneo o di pietra, come nel ḩānqā al-Farāfra ${ }^{75}$.

Terry Allen nota che la suddivisione di un arco tramite un architrave (o, in altri casi, l'utilizzo di un architrave quale copertura di una porta e base per la finestra ad arco soprastante), sia esso di pietra o ligneo, è ricorrente nell'architettura ayyubide e che le differenze di materiale e forma sono dovute a varianti locali, con una prevalenza dell'uso

67 La madrasa fu costruita tra il $1172-73$ e il 1223 , ma la facciata sulla corte con le travi lignee appartiene probabilmente ai lavori più recenti (Sauvaget - Écochard - Sourdel-Thomine 1938-1950, 84-85, tavv. XVIIIXIX; Tabbaa 1997, fig. 117; Allen 1999, cap. 2).

68 Del 1167-68 (Allen 1999, cap. 4, fig. 19).

69 Corte del 1219 (Tabbaa 1997, figg. 150, 152).

70 Del 1238 (Tabbaa 1997, 164-168, figg. 147, 184 pianta, 185-188). Da notare inoltre che una seconda trave in legno è inserita al di sotto della principale all'interno di fessure rettangolari della pietra (Tabbaa 1997, fig. 186). Nel cortile un architrave ligneo corre lungo tutti gli elevati all'altezza superiore delle porte (Allen 1999, cap. 8; si riferisce all'edificio come "Khânaqâh of Dayfa Khâtûn"). Un altro architrave, anch'esso continuo ma forse non originale, corre sulle finestre del piano superiore, sovrastato da archi ribassati; tali archi sono definiti da Allen (Allen 1999, cap. 8) "slightly pointed segmental arches", mentre in Tabbaa (1997, fig. 187) sono disegnati come archi a tutto sesto ribassato; a giudicare dalle immagini del monumento (Allen 1999, fig. 59, Tabbaa 1997, fig. 188) e considerando la possibilità che la parte superiore dell'edificio abbia subito modifiche per un restauro (come ipotizzato da Allen per la presenza dell'architrave), ci sembra più probabile che si tratti di archi leggermente acuti.

71 Tabbaa 1997, fig. 178.

72 Tabbaa 1997, fig. 62.

73 Allen 1999, cap. 8; Tabbaa (1997, 164-182) non sembra essere della stessa opinione.

74 Tabbaa 1997, fig. 178

75 Tabbaa 1997, figg. 185-188. Forse anche la madrasa al-Firdaws aveva una conformazione simile, sebbene le travi lignee siano oggi perdute (Tabbaa 1997, figg. 207-209). 
del legno nella Siria meridionale e al Cairo $^{76}$, mentre Sauvaget vedeva nell'uso del legno, che indebolisce la struttura degli edifici costruiti in pietra, la persistenza di una pratica dovuta all'influenza dei metodi costruttivi di Mesopotamia e Iran, dove infatti il materiale da costruzione prevalente erano i mattoni ${ }^{77}$. A prescindere dalle ipotesi sull'origine dell'uso di travi lignee, sia che si tratti di tradizioni locali sia che si tratti di influenze esterne, gli esempi citati dimostrano che era una pratica costruttiva consolidata in tutto il territorio ayyubide nel XII e XIII secolo. Un'ulteriore conferma viene dal fatto che le travi lignee utilizzate in questi edifici sostengono archi di tipi diversi - uno solo dei quali, tra l'altro, del tipo impiegato a Shawbak.

A Shawbak, dunque, si utilizzò un tipo di arco, quello a tutto sesto ribassato integrale degli $\bar{\imath} w \bar{a} n$, che non è affatto comune nell'architettura ayyubide ${ }^{78} \mathrm{e}$ il cui utilizzo rinvia sicuramente ad una diffusione locale, vista la ricorrenza di archi non troppo dissimili ad 'Ajlun; la possibilità che la maggior parte degli archi ribassati a Shawbak fosse sostenuta da travi lignee, invece, li riporta all'interno della tradizione costruttiva ayyubide tra la seconda metà del XII e la prima metà del XIII secolo, con una particolare affinità con alcune caratteristiche di due edifici aleppini degli anni '30 del XIII secolo, la madrasa al-Firdaws e il hhānqā al-Farāfra; infine, l'impiego dei due elementi combinati (arco ribassato/trave lignea) trova un unico parallelo nella madrasa al-Firdaws. La diffusione a livello locale dei due elementi combinati è dimostrata dalla presenza di fori adatti ad ospitare travi lignee nella sala di ricevimento di $\operatorname{Karak}^{79}$.

\subsection{Le nicchie e il sistema di ventilazione}

Negli edifici residenziali/palatini di periodo ayyubide la presenza di nicchie è rara e in nessun caso queste sono impiegate in maniera regolare e sistematica come a Shawbak: l'unico esempio a noi noto è nel palazzo della cittadella di Aleppo ${ }^{80}$. Al contrario sembrano essere abbastanza comuni nelle madrasa di Aleppo e Damasco nella prima metà del XIII secolo, con funzione di vano contenitore/armadio per i libri e talvolta corredate, come a Shawbak, da un'intercapedine per la ventilazione. Tuttavia solo nella madrasa al-Firdaws ${ }^{81}$

76 Allen 1999, cap. 1. Non sempre, tuttavia, gli incavi servivano per l'inserimento di architravi: nella madrasa al-Kāmilīyya di Aleppo (1230-37), dove sono visibili gli incavi per le travi, si trattava di cornici lignee per porte o chiusure dei passaggi (Allen 1999, cap. 8, fig. 29). Per il Cairo citiamo il mausoleo dell'Imām al-Šăfi ‘'i (Creswell 1959, II, fig. 31, tavv. 22b, 68), dove la trave lignea è intagliata e contiene un'iscrizione.

77 Sauvaget - Écochard - Sourdel-Thomine 1938-1950, 22-25. Tra i primi esempi in Siria la Qubba al-Tawāwis a Damasco (1103-04); l'edificio, che venne distrutto nel 1938, era costruito in parte in mattoni (Sauvaget Écochard - Sourdel-Thomine 1938-1950, 1-13; Allen 1999, cap. 1)

78 Brown (1988a, 50) nota che i piccoli archi laterali sono inusuali, «more rounded than the typical pointed segmental arches of the Ayyubid period», non particolarmente diagnostici e senza confronti.

79 In una torre a Burzey si trovano archi a tutto sesto ribassato poggianti su architravi in pietra (Dangles et al. 2001, fig. 12)

80 Ai lati dell' '̄wān nord e di quello ovest della corte grande e in altri ambienti a nord est e est (Tabbaa 1997 figg. 31, 44). Una sola nicchia, particolarmente profonda, si affaccia sulla corte a Qal'at Șahyūn, ma forse si trattava di un passaggio (Tabbaa 1997, fig. 56).

81 Tabbaa 1997, figg. 192-194, 202. 
e, in misura minore, nella madrasa al-Kāmīliyya, forse da attribuire allo stesso architetto ${ }^{82}$, nonché nell'ambiente cupolato d'angolo inserito nella seconda fase costruttiva del Mašhad al-Husayn di Aleppo ${ }^{83}$, e nel Bīmāristān al-Qaymarī a Damasco (Șāliḥīyya) ${ }^{84}$ le nicchie sono utilizzate estensivamente e con una disposizione tripartita che può ricordare l'impianto di Shawbak ${ }^{85}$.

Le intercapedini verticali presenti sul retro delle nicchie nell'ambiente UF1 a Shawbak, con la funzione di elementi per la ventilazione e lo scambio di aria calda/fredda, sembrano essere state molto diffuse negli edifici ayyubidi ${ }^{86}$. Il loro impiego ha infatti una lunga tradizione nell'architettura residenziale islamica e preislamica ${ }^{87}$ : denominati "cattura vento"

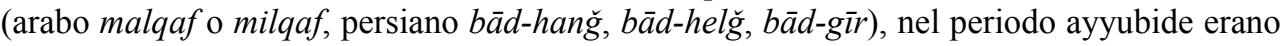
un elemento comune nella maggior parte delle case egiziane ${ }^{88}$, e sono attestati in alcuni edifici, anche religiosi, sia egiziani sia siriani. Possiamo citare gli ambienti di ricevimento nella cittadella di Aleppo (corte maggiore) e a Qal'at Nağm ${ }^{89}$; ad Aleppo le madrasa alFirdaws $^{90}$, al-Ṣulțāniyya ${ }^{91}$, al-Šarafìyya ${ }^{92}$, e a Damasco il Bīmāristān al-Qaymarị ${ }^{73}$. Mentre

82 L'edificio è del 1230-1237 (Tabbaa 1997, figg. 114-115); Allen (1999, cap. 8, fig. 28) ipotizza che sia stata costruita dallo stesso architetto della al-Firdaws.

83 Fase databile tra il 1216 e il 1237 (Tabbaa 1997, 120, figg. 88, 89b) o al 1231-1236 (Korn 2004, II, 218-219, 237-238, fig. 41).

84 Iniziato nel 1248, completato pochi anni dopo (Herzfeld 1946, fig. 53:646; Allen 1999, cap. 10).

85 Citiamo anche l'uso estensivo delle nicchie nell' $\bar{w} w \bar{a} n$ nord della madrasa "Ā Ailīyya ad Aleppo (Sauvaget Écochard - Sourdel-Thomine 1938-1950, 84-85, tavv. XVIII-XIX; Tabbaa 1997, 138, fig. 117; Allen 1999, cap. 2).

86 In molti edifici palatini il rinfrescamento degli ambienti era ottenuto anche tramite percorsi d'acqua che confluivano in una vasca al centro della sala delle udienze; solitamente i percorsi d'acqua prendevano inizio da uno šădīrwān (un pannello inclinato e intagliato su cui veniva fatta scorrere l'acqua). Gli esempi preservati sono quelli dei palazzi della cittadella di Aleppo e di Diyarbakr, entrambi con šădīrwān e vasca ottagonale al centro della corte, mentre a Qal'at Șahyūn e Qal'at Nağm si preserva la vasca centrale ma non lo šădìr wān, di cui probabilmente erano forniti. Qașr al-Banāt e il Mațbah al-'Ağamī avevano una vasca centrale. Ibn Šaddād (citato in Tabbaa 1997, 95) afferma che solo dopo la costruzione del palazzo della cittadella di Aleppo divenne comune ad Aleppo la pratica di collocare nelle case una fontana al centro della corte; di fatto la maggior parte degli edifici palatini appartenuti al principato di Aleppo (oltre che Qașr al-Banāt e Diyarbakir) presentano un bacino (ottagonale, polilobato o circolare) al centro della sala (spesso una corte aperta).

87 Lézine $(1972 b, 10)$ fa risalire l'uso di malqaf in Egitto all'epoca faraonica; anche alcune case di Fustat erano provviste di malqaf (Lézine 1972a, 86).

88 Molte erano le modalità di rinfrescamento dell'aria utilizzate in Egitto (Lézine 1971, 12-13); tra queste la più semplice è descritta nel XVI secolo da Leone l'Africano come «une haute cheminée qui a une ouverture au sommet et une autre à la base... le vent entre par le haut, sort par la base et amène un peu de fraicheur» (citato in Lézine 1971, 12) e sembra essere del tipo utilizzato a Shawbak. In Egitto il malqaf più diffuso sembra essere un tipo costituito da una specie di tettoia sul tetto con griglia nell'ambiente sottostante.

89 Tabbaa 1997, 90.

90 Allen (1999, cap. 8) individua tre intercapedini su ciascun lato dell'īwān nord sulla corte, due su ciascuno dei lati dell' '̄wān nord esterno, ma a giudicare dalla pianta (Tabbaa 1997, fig. 193) su quest'ultimo ìwān si trova un'intercapedine su ciascun lato. Tabbaa $(1997,169)$ propone che le nicchie fossero impiegate «probably for the storage of books».

91 Allen, che pure non esclude che possano essere state armadi, individua intercapedini nelle nicchie sul muro qiblī della sala di preghiera e nei due recessi della sala funeraria («now closed with shutters» [Allen 1999, cap. $8])$. 
gli esempi siriani sono del tipo rinvenuto a Shawbak ${ }^{94}$, al Cairo, invece, gli elementi per la ventilazione che si trovano nelle madrasa o nelle moschee sono di tipologia diversa ${ }^{95}$. 'Abd al-Lațîf al-Bağdādī, che visitò il Cairo intorno al 1200, descrisse l'abilità con cui queste strutture erano costruite, i costi, che potevano arrivare a cifre consistenti, e l'esposizione sempre a settentrione ${ }^{96}$.

\subsection{Decorazione}

Gli ambienti che formavano il complesso della sala delle udienze si presentano oggi completamente spogli di ogni decorazione, benché almeno l'alloggiamento rettangolare che corre lungo le pareti settentrionali della sala maggiore dovesse ospitare un inserto. L'ipotesi che fosse un elemento ornamentale è suffragata dall'altezza della cavità nel muro, insufficiente, ad esempio, se si fosse trattato di un alloggio per travi lignee, e dal confronto con cornici lignee ornamentali preservatesi in situ. Esempi di cornici lignee, tuttavia, si trovano negli edifici di epoca ayyubide solo in Egitto e non corrispondono esattamente al caso di Shawbak, trattandosi sempre di fasce ornamentali inserite nelle pareti ad un'altezza maggiore (sopra le porte o sotto il tamburo della cupola), come nel mausoleo dell'imām Shāfi ‘ $\mathfrak{i}^{97}$ e in quello di Šağarat al-Durr ${ }^{98}$, entrambi al Cairo. Le molte cornici in legno conservate nei musei, spesso con dimensioni simili a quelle registrate a Shawbak (altezza circa $10 \mathrm{~cm}$ ), sono datate al periodo fatimide - con scene con raffigurazioni antropomorfe e zoomorfe, oltre che ornati vegetali ed elementi iscritti99 - e anche all'epoca ayyubide durante la quale si prediligeva la riproduzione di iscrizioni e di ornati vegetali ${ }^{100}$.

92 Del 1205, ma quasi tutto l'edificio è di circa 20 anni dopo; Sibt b. al-'Ağamī (XV secolo) riferisce di un $b \bar{a} d-$ hanğ nell'̄iwān (Allen 1999, cap. 8).

93 «Air shafts reported by Wulzinger and Watzinger» nella cucina (Allen 1999, cap. 10)

94 Ci riferiamo a quelli nelle madrasa siriane, poiché non abbiamo informazioni per quelli nei palazzi di Aleppo e Qal'at Nağm

95 Del tipo con tettoia sul tetto dell'edificio e griglia che si apre sull'ambiente interessato. Gli esempi più antichi preservatisi sono quelli nella cappella copta di Dār al-Banāt, datata all'inizio dell'XI secolo (Lézine 1971, 15, n. 3; Lézine 1972a, 74, 79); altri esempi sono nella moschea di al-'Azhar (ma probabilmente recenti come tutto il tetto), nella moschea di al-Sālih Talā'̄'‘ (del 1160); nella madrasa al-Kāmilīyya (del 1225, del tipo a tettoia e con un "camino" profondo 3,75 m) e nella madrasa di al-Nāsir Muhammad (del 1303-1304). Le tettoie sono tutte scomparse (Creswell 1959, I, 45, 284-285; II, 80-82).

96 Citato in Lézine $(1971,7$, n. 2).

97 Del 1211 (Creswell 1959, II, 68; Behrens-Abouseif 1989, 87).

98 Si tratta di una cornice lignea che corre lungo tutta la superficie interna dell'ambiente, sopra gli ingressi. L'edificio è del 1250, ma «the wooden frieze running along the walls with carved inscriptions and arabesques is Fatimid in style and must have belonged to an earlier building» (Behrens-Abouseif 1989, 92).

99 Tra i pannelli più noti sono quelli con scene animate provenienti probabilmente dal palazzo fatimide di alMustanșir al Cairo, rinnovato tra il 1058 e il 1065 (Pauty 1931, 48-50, nn. 3465-3473, 4063, tavv. XLVILVIII).

100 Tra gli esempi di epoca ayyubide un pannello con ornato vegetale al Museo Benaki di Atene (inv. 9242 attribuito all'Egitto tra la fine del XII e l'inizio del XIII secolo, altezza $12 \mathrm{~cm}$, conservato per $181 \mathrm{~cm}$ Delpont - Fauret - Koïkas 2001, 46, n. 35,) e uno con iscrizione benaugurale (in corsivo su tralcio foliato) al Metropolitan Museum di New York (inv. 30.112.3). Quest'ultimo conserva tracce di policromia (rosso e blu) e di doratura originari (attribuito all'Egitto o alla Siria al XIII secolo, altezza 11,4, conservato per 119,4 cm; Heilbrunn Timeline of Art History, 2000; Delpont - Fauret - Koïkas 2001, n. 36, 46). 
I palazzi ayyubidi dell'area siro-palestinese a noi noti erano decorati con ricchi portali, spesso con elementi a intarsio, 'ablaq e muqarnas, che si ritrovano spesso anche negli ambienti interni, dove un importante contributo decorativo era apportato dall'uso coreografico dell'acqua (convogliata in canalette, vasche ornamentali, šadīrwān) e talvolta dagli ornati geometrici dei pavimenti ${ }^{101}$. Nessuno di questi elementi è stato finora rinvenuto a Shawbak, che sembra invece essere l'unico caso di edificio palatino che possedeva una fascia decorata inserita sui muri della sala maggiore del complesso delle udienze.

\subsection{Coperture degli ambienti: a crociera ribassata, a botte con uncini, a botte}

L'ambiente UF1 a Shawbak, la sala centrale della $q \bar{a}$ ' $a$, era coperto ${ }^{102}$ o da una volta a crociera ribassata o da una volta a crociera corredata di ampio impluvium centrale per garantire la luminosità dell'ambiente.

Pochi sono gli esempi a noi noti di volta a crociera ribassata in Siria, mentre non è infrequente nei pochi monumenti attribuiti al XIII secolo a Baghdad, tutti però costruiti in mattoni cotti: si ritrova, infatti, in tutti gli ambienti ai lati della corte del cosiddetto palazzo abbaside, oggi ritenuto una madrasa ${ }^{103}$, nonché nei due ambienti rettangolari adiacenti all'ingresso nella madrasa al-Mustanșirīyya ${ }^{104}$. Quanto alla volta a crociera con impluvium centrale non mancano in costruzioni in pietra siriane: ad esempio ad Aleppo, in un ambiente del Mašhad al-Dikka ${ }^{105}$ e nel Mašhad al-Husayn ${ }^{106}$; particolarmente significativo è il confronto con la sala di ricevimento nella cittadella di Bosra ${ }^{107}$.

La volta a crociera con uncini, che costituiva probabilmente la copertura dell'ambiente UF2 di Shawbak (fig. 6) ${ }^{108}$, è piuttosto comune negli edifici siriani ed egiziani nel periodo ayyubide e mamelucco ${ }^{109}$.

101 Come ad esempio ad Aleppo (Tabbaa 1997, fig. 31). I sondaggi di scavo effettuati in UF1 e in UF2 non hanno messo in luce nessuna evidenza del pavimento originario, né dello strato di allettamento dello stesso; in altri ambienti esterni al complesso di ricevimento, invece, le tracce rinvenute su uno strato di intonaco pavimentale e le poche lastre rinvenute in situ indicano dei pavimenti lastricati, alcuni in marmo, altri in pietra calcarea (Brown 1988a, 77, 86, 88, 124-125; 1988b, 232).

102 Il fatto che la $q \bar{a}$ ' $a$ sia coperta segue un'evoluzione evidenziata da Sayyid (1987, 51; nella sua più ampia argomentazione del passaggio dal mağlis all'īwān nel Cairo ayyubide): erano prima corti aperte, ma la crescente mancanza di spazio urbano fa sviluppare in verticale le strutture architettoniche, insieme al restringimento della durqā' $a$ che rende più facile la sua copertura.

103 Costruito intorno al 1230 (Herzfeld 1942, 29, fig. 19: «almost horizontal, cloister vaults, which reveal great virtuosity in vaulting»).

104 Del 1227-1234 (Creswell 1959, II, fig. 68).

105 L'ambiente risale al 1146 (Sauvaget 1928, fig. 9; Tabbaa 1997, 109, fig. 82).

106 Iniziato nel 1174 (Tabbaa 1997, 111, fig. 88).

107 Abel 1956, 131; Tabbaa 1997, fig. 65; Korn 2004, II, 176, fig. 32.

108 «The central chamber supported a particular style of cloister vault that has been called a 'mitered' vault. This vaulting in the central chamber probably supported a square compluvium at the top» (Brown 1988a, 50).

109 Uno degli esempi più antichi è la volta del portale della madrasa al-Ḥalāwīyya ad Aleppo (1149), ma si riscontra anche nell' '̌w $w \bar{a} n$ est del bìmaristān di Damasco; nella «eastern door [...] , to a vaulted passageway along the southern side» del Hān Gümrök a Urfa (tardo XII secolo); in un ambiente nella cittadella di Gaziantep; ad Aleppo: nell'̄iwān e nei vani laterali della sala di preghiera della madrasa al-Šādbahtīyya (1193), nell' '̄wān di ingresso della Zāhirīyya (completata nel 1216-1217), nel portale e nei vani laterali della sala di preghiera della Șultānīyya (completata nel 1225), nell'īwān esterno della al-Firdaws (1235), nei due 
Quanto alle volte a botte sono estremamente comuni nell'architettura di periodo ayyubide, in particolare per gli ambienti di minore importanza (corridoi, grandi sale rettangolari etc.).

\subsection{Finitura con nastrino}

La maggior parte delle finiture riscontrate nel complesso palatino presenta un nastrino decorativo ottenuto con martellina dentata $(\mathrm{S} 6)^{110}$. Nastrini simili si riscontrano nelle finiture di moltissimi edifici di epoca ayyubide, soprattutto a Damasco, quasi tutti collocabili nel periodo tra l'ultimo quarto del XII e la prima metà del XIII secolo ${ }^{111}$.

\section{IPOTESI DI DATAZIONE E CONCLUSIONI}

L'unitarietà del progetto architettonico del complesso di ricevimento di Shawbak autorizza ad attribuirlo ad un unico intervento costruttivo iniziale ${ }^{112}$. L'analisi degli elementi architettonici consente di collocarlo nella tradizione architettonica ayyubide, pur senza riuscire a circoscrivere ad un periodo più preciso il momento della sua costruzione che non viene indicata in alcuna delle fonti storiche a noi note; sebbene, inoltre, nessun elemento architettonico possa essere attribuito esclusivamente al periodo ayyubide, una simile datazione è verosimile per le evidenze archeologiche dello scavo di R. Brown. L'unico edificio che presenta una similarità quasi pedissequa nell'impianto architettonico, la $q \bar{a}$ ' $a$ del complesso palatino di Karak, non è di grande aiuto per una datazione più precisa, poiché quasi certamente successivo (cfr. n. 33).

Sicuramente il 1189, l'anno in cui la guarnigione crociata di Shawbak si arrese al lungo assedio inflittole da Salāḥ al-Dīn, deve essere considerato quale terminus post quem per la

vani ai lati della cupola del mihrāab della Šarafiyya (tra il 1198 e il 1234) e nell'arco meridionale trilobato del Masğid al-Ğāmi‘ al-Bāb (Allen 1986, 14, 82-83, fig. 24).

110 M. Nucciotti (2007, 44-45; in stampa, 4-5) rileva che nel prospetto meridionale esterno della sala è presente un tipo diverso di finitura (S1), impiegata in lavori di ristrutturazione successivi (cfr. nn. 112, 117).

111 A Damasco: porte della grande moschea, Bīmāristān di Nūr al-Dīn, madrasa di Nūr al-Dīn, Bāb al-Sagī̄r, turba Sitt al-Sām al-Sug̉rā, turba al-Nağmīyya, madrasa al-“Ādilīyya, madrasa al-Farrūhšāhīyya, madrasa al-Šāmīyya, madrasa al-Ruqnīyya extra muros, turba al-Farnathīyya, Madrasa al-'Izzīyya, madrasa alSāhiba, turba Amat al-Latīf, turba al-Qaymarī, madrasa al-Ğahārkasīyya, porta est della cittadella; ad Aleppo: madrasa al-Sultānīyya, Maqām Ibrāḥ̄m superiore, blocco di ingresso alla cittadella; portale di Ibn Turayra; infine, portale nord e cittadella di Bosra e portale della cittadella di Palmira (Allen 1999, cap. 1-10). Il più antico di questi edifici (Palmira) data al 1133, tutti gli altri datano tra l'ultimo quarto del XII e la prima metà del XIII secolo. In quasi tutti i casi citati i nastrini sono lavorati con strumenti dentati; Allen (1999, cap. 2) afferma che l'uso di utensili non dentati per i nastrini è più frequente negli edifici damasceni dell'inizio del XII secolo.

112 Nel complesso palatino sono stati individuati interventi successivi alla sua costruzione. Nel corso dello scavo R. Brown (1988b, 240-242) ha individuato due fasi ayyubidi e una terza fase, di ristrutturazione e rioccupazione del complesso di ricevimento, che posiziona intorno al 1260 (anticipata al tardo periodo ayyubide sulla base dei ritrovamenti ceramici di importazione da Milwright [2006, 22-23]). Vannini Nucciotti ([eds.] 2007, 18, 22) e Nucciotti (2007, 44-45; in stampa, 4-5) individuano una seconda fase ayyubide, di ristrutturazione, dell'edificio, durante la quale la sala principale venne collegata direttamente con gli ambienti del hammām; tale ristrutturazione viene attribuita a al-Mu'azzam 'Isa dopo il terremoto del 1212 sulla base dell'analisi stratigrafica degli elevati (cfr. nota 110: le finiture rinvenute [S1], sono «assimilabili a quelle in uso a Damasco tra l'inizio e la metà del XIII secolo» e «riferibili all'intervento dei maestri damasceni di Al-Mu'azzam attivi nelle ricostruzioni successive al sisma del 1212» [Nucciotti in stampa, 4-5]). 
costruzione del complesso di ricevimento; non è improbabile che sia avvenuta nel lungo periodo, dal 1197 al 1226, in cui la cittadella era annoverata tra i possedimenti di alMu'azzam 'Isa (formalmente signore di Shawbak dal 1208). Ciò troverebbe riscontro nel racconto quasi coevo di Ibn Šaddād (1145-1234), che riferisce delle attività costruttive di tale personaggio, il quale avrebbe fortificato e abbellito (hașsanahu wa hassanahu) Shawbak, impiantandovi giardini (al-ašğăr min sā'ir al-aqtāar, alberi di ogni regione) che giunsero ad essere paragonati quanto a bellezza con quelli di Damasco. ${ }^{113}$ Il violento terremoto del 1212, che arrecò ingenti danni anche a Shawbak, potrebbe essere individuato quale più preciso terminus post-quem. A sostegno di questa datazione è il riscontro particolarmente pertinente con l'ambiente di ricevimento del donjon di Bosra del 1213-14, con cui Shawbak condivide sia la composizione tripartita/mağlis sia la presenza della volta a crociera con impluvium centrale.

La costruzione del complesso potrebbe tuttavia essere collocata in un periodo successivo a al-Mu'azzam 'Isa, che nel 1226 avrebbe ordinato, secondo Ibn Furāt (m. $1405)^{114}$, la distruzione di Shawbak per esigenze militari - si suppone solo delle fortificazioni e non delle costruzioni residenziali. Gli ultimi signori ayyubidi di Shawbak commissionarono sicuramente attività edilizie sulla cittadella, come è testimoniato da alcune epigrafi rinvenute sul sito. Tra queste una in cui viene menzionato il protocollo del sultano al-Kāmil (in possesso di Shawbak con vicende alterne dal 1229 al 1238$)^{115}$ e una seconda che attesta la fine dei lavori di costruzione di torri e mura voluti dal sultano alMalik al-Șāliḥ Nağm al-Dīn nell'anno 643/1245-46 ${ }^{116}$. A sostegno di una datazione al secondo quarto del XIII secolo possono essere annoverati il riscontro con certe caratteristiche architettoniche della madrasa al-Firdaws del 1237, o tra il 1235-36 e il 1241 (la tripartizione con cortina tripartita/mağlis delle $q \bar{a}^{\prime} a$ residenziali, gli archi ribassati con architravi lignei) e del ḩānqā al-Farāfra del 1238 (la tripartizione con cortina tripartita/mağlis della $q \bar{a}^{\prime} a$ residenziale e gli architravi lignei sui pilastri) ${ }^{117}$

La struttura architettonica del complesso di ricevimento di Shawbak riflette sicuramente il quadro culturale e sociale in cui agivano i signori ayyubidi. Mentre i castelli crociati erano postazioni militari a difesa e controllo del territorio circostante, secondo le modalità europee dell'incastellamento, dove era la funzione militare a decretare le caratteristiche generali della vita quotidiana e conseguentemente dell'architettura, nei complessi palatini

113 Ibn Šaddād 1963, 80. Vedi Korn (2004, 160) per una lettura critica dell'espressione hașsanahu wa hassanahu, vero topos letterario nelle fonti storiche. Per R. Brown (1988b, 240-242) la costruzione del complesso deve essere attribuita proprio a al-Mu'az̧zam 'Isa.

114 Ibn al-Furāt, citato in Brown (1988a, 18).

115 L'iscrizione non proviene da un contesto archeologico ed è mutila della prima parte; si trattava probabilmente di un testo di costruzione (Korn 2004, 93 n. 41; Dotti 2009, 117: lastra epigrafica 50. Dotti 2010, 28). Già R. Brown (1988a, 20) aveva ipotizzato che il sultano al-Kāmil avesse restaurato e ampliato edifici a Shawbak.

116 Korn 2004, 93 n. 42; Dotti 2009, 117: lastra epigrafica 49. Dotti 2010, 29. Menzioniamo anche un'altra epigrafe ritenuta provenire dalla cittadella, ma rinvenuta all'inizio del XX secolo sulla porta dell'adiacente maqām di Sulaymān al-Dirānī, recante la data 646/1248 (Brown 1988a, 20; Korn 2004, 93, n. 43).

117 Per M. Nucciotti (2007, 45; in stampa, 4-5) l'assenza di finiture di tipo S1 nella sala di ricevimento e la loro comparsa, invece, nella ristrutturazione del prospetto meridionale esterno (attribuita a dopo il terremoto del 1212; cfr. nn. 110, 114), prova che la costruzione del complesso debba essere precedente al 1212. 
ayyubidi la struttura militare e quella urbana circondavano e proteggevano il nucleo residenziale destinato al governante, la cui condotta non voleva discostarsi da quella dei signori che risiedevano nelle grandi città del sultanato. L'architettura del complesso di ricevimento di Shawbak, sobria e dalle dimensioni contenute, enfatizza con la progressione crescente dei volumi e delle linee di fuga il ruolo di rappresentanza dell'ambiente, alleggerito nella sua austerità solo dalla fascia decorativa in legno. L'accesso diretto all'area del hammām, introdotto in un secondo momento, fa emergere l'aspetto residenziale-conviviale della vita dei signori ayyubidi a Shawbak.

L'elaborata progettazione architettonica, evidenziabile nella disposizione planimetrica e nelle coperture delle sale - ciascuna differente per forma della volta ed elevazione -, è forse inattesa in una costruzione apparentemente provinciale e sembra indicare una committenza di un certo rango e con precise esigenze di auto-rappresentazione. Bisogna tenere conto della particolare configurazione "confederale" dello Stato in questo periodo, e smussare la contrapposizione inevitabile tra siti urbani (quali Aleppo, il Cairo e Damasco) e insediamenti provinciali. Gli Ayyubidi concepivano il potere come un affare di famiglia, in cui ciascun rappresentante aveva diritto ad esercitarlo nei propri territori ${ }^{118}$ e la cultura che esprimevano era, oltre che militare, sentitamente urbana: la costruzione di un edificio rappresentativo come quello cui doveva appartenere il complesso di ricevimento è pertanto perfettamente comprensibile anche in una località relativamente minore come Shawbak.

\section{BIBLIOGRAFIA}

ABEL, E.

1956 La citadelle ayyubite de Bosra Eski Cham: Annales archéologiques de Syrie VI (1956),

ALLEN, T. pp. $95-138$

1986 A Classical Revival in Islamic Architecture, Wiesbaden 1986.

1999 Ayyubid $\quad$ Architecture, California 1999

ASLANAPA, O (www.sonic.net/ tallen/palmtree/ayyarch/index.htm)

1962 Erster Bericht über die Ausgrabungen des Palastes von Diyarbakir: Istanbuler Mitteilungen 12 (1962), pp. 115-128.

BEDDEK, K.

2001 Le complexe ayyubide de la citadelle de Șalāh al-Dīn: bain ou palais?: Archéologie Islamique 11 (2001), pp. 75-90.

BEHRENS-ABOUSEIF, D

1988 The Citadel of Cairo: Stage for Mamluk Ceremonial: Annales Islamologiques 24 (1988), pp. 25-79.

1989 Architecture of the Ayyubid Period: D. BEHRENS-ABOUSEIF, Islamic Architecture in Cairo. An Introduction, Leiden - New York 1989, pp. 78-93.

BLOOM, J.M.

1987 The Mosque of the Qarafa in Cairo: Muqarnas 4 (1987), pp. 7-20.

118 Humphreys 1977, 10-11. Lo storico utilizza le definizioni di sovranità collettiva e di confederazione familiare. 
BROWN, R.M.

1988a Report of the 1986 Excavations at Shobak, 1988, unpublished report submitted to the Dr. Adnan Hadidi, Director General of Antiquities (ACOR Amman).

1988b Late Islamic Shobak: A Summary Report of the 1986 Excavations: Annual of the Department of Antiquities of Jordan XXXII (1988), pp. 225-245.

1989 Excavations in the Fourteenth-Century Mamluk Palace at Kerak: Annual of the Department of Antiquities of Jordan XXXIII (1989), pp. 287-304.

CRESWell, K.A.C.

1959 The Muslim Architecture of Egypt, Oxford 1959.

DANGLES, P. ET ALII

2001 Bourzë̈. Hișn Burzaīh/ Burzūīh/ Qal'at Mirzé. Rapport de la mission effectuée du 27/05/2001 au 02/06/2001 (www.castellorient.fr/0-Accueil/indexfran.htm).

DAVID, J.-C.

2007 Ayyubid palace architecture in Syria: S. BiANCA (ed.), Syria. Medieval Citadels Between East and West, Torino 2007, pp. 51-70.

DELPONT, E. - FAURET, A. - KoÏKAS, Y.

2001 L'Orient de Saladin: l'art des Ayyoubides: exposition présentée à l'Institut du monde arabe, Paris, du 23 octobre 2001 au 10 mars 2002, Paris 2001

DOTTI, F

2009 49. Lastra epigrafica; 50. Lastra epigrafica: G. VANNINI - M. NuCCIOTTI (eds.), Da Petra a Shawbak, Archeologia di una frontiera, Firenze 2009, p. 117.

2010 Qal'at al-Shawbak: an interpretation on the basis of the epigraphic data: P. MATTHIAE - F. PinNOCK - L. Nigro - N. MARChetTI (eds.), Proceedings of the $6^{\text {th }}$ ICAANE Congress, Rome 5 May - 10 May 2009 [sic; 2008], "Sapienza”, Università di Roma, III, Wiesbaden 2010 , pp. 23-36.

FEÏSS-JEHEL, C. ET ALII

2008 La forteresse de Sadr, une lecture géo-historique au temps des croisés: EchoGéo 4 (2008) (http://echogeo.revues.org/index2228.html).

GARCIN, J.C

2001 Evolution urbaine et architecture au temps des Ayyoubides: L'Orient de Saladin: l'art des Ayyoubides: exposition présentée à l'Institut du monde arabe, Paris, du 23 octobre 2001 au 10 mars 2002, Paris 2001, pp. 72-83.

GELICHI, S.

2006 The Citadel of Hārim: H. KENNEDY (ed.), Muslim Military Architecture in Greater Syria. GOITEN, S.D.

From the Coming of Islam to the Ottoman Period, Leiden 2006, pp. 184-199.

1977 A Mansion in Fustat: a Twelfth Century Description of a Domestic Compound in the Ancient Capital of Egypt: H. Miskimin ET ALII (eds.), The Medieval City, New Haven 1977, pp. $163-178$

GONNELLA, J.

2006 The Citadel of Aleppo: recent studies: H. KenNedy (ed.), Muslim Military Architecture in Greater Syria. From the Coming of Islam to the Ottoman Period, Leiden 2006, pp. 165175.

2007 Introduction to the Citadel of Aleppo: S. BIANCA (ed.), Syria. Medieval Citadels Between East and West, Torino 2007, pp. 103-138.

HEIDEMANN, $\mathrm{S}$.

2006 The Citadel of al-Raqqa and Fortifications in Middle Euphrates Area: H. KENNEDY (ed.), Muslim Military Architecture in Greater Syria. From the Coming of Islam to the Ottoman Period, Leiden 2006, pp. 122-150. 


\section{HEILBRUNN TIMELINE OF ART HISTORY}

2000 Panel [Egypt or Syria] n. inv. 30.112.3: Heilbrunn Timeline of Art History, The Metropolitan Museum of Art (www.metmuseum.org/toah/ho/07/nfe/ho_30.112.3.htm), New York 2000.

HERZFELD, E.

1942 Damascus: Studies in Architecture - I: Ars Islamica IX (1942), pp. 1-53.

1946 Damascus: Studies in Architecture - III: Ars Islamica XI-XII (1946), pp. 1-71.

1954-1956 Matériaux pour un Corpus Inscriptionum Arabicarum, pt. 2, Syrie du Nord. Inscriptions et monuments d'Alep (Mémoires de l'Institut Français d'Archéologie Orientale), Le Caire 1954-1956.

HiLlENBRAND, R.

1985 Eastern Islamic Influences in Syria: Raqqa and Qal'at Ja'bar in the Later $12^{\text {th }}$ Century: J. RABY (ed.), The Art of Syria and the Jazira, 1100-1250, Oxford 1985, pp. 21-48.

1994 Islamic Architecture: Form, Function and Meaning, Edinburgh 1994.

HUMPHREYS, S.

1977 From Saladin to the Mongols. The Ayyubids of Damascus, 1173-1260, New York 1977.

IBN ŠADDĀD

1963 Liban Jordanie Palestine Topographie Historique d'Ibn Šaddād, éd. critique d'al-A 'lāq al-hatīira: S. DAHAN (ed.), Damas 1963.

KORN, L.

2004 Ayyubidische Architektur in Ägypten und Syrien. Bautätigkeit im Kontext von Politik und Gesellschaft 564-658/1169-1260 (Abhandlungen des Deutschen Archäologischen Instituts Kairo, Islamische Reihe 10), Heidelberg 2004.

2008 The Sultan stopped at Halab. Artistic exchange between Syria and Iran in the late $5^{\text {th }} / 11^{\text {th }}$ century: L. KORN - E. ORTHMANN - F. SCHWARTZ (eds.), Die Grenzen der Welt. Arabica et Iranica ad honorem Heinz Gaube, Wiesbaden 2008, pp. 105-121.

LÉZINE, A.

1971 La protection contre la chaleur dans l'architecture musulmane d'Egypte: Bulletin d'Etudes Orientales XXIV (1971), pp. 7-17.

1972a Les salles nobles des palais mamelouks: Annales Islamologiques 10 (1972), pp. 63-148.

1972b Persistance de traditions pré-islamiques dans l'architecture domestique de l'Égypte musulman: Annales Islamologiques 11 (1972), pp. 1-22.

MichAUDEL, B.

2001 Le chateau de Saône (Sahyûn, Qal'at Salâh al-Dîn) et ses defences: Archéologie MiLWRIGHT, M Islamique 11 (2001), pp. 201-206.

2006 Southern and Central Jordan in the Ayyubid period: Archaeological and historical perspectives: Journal of the Royal Asiatic Society 16.1 (2006), pp. 1-27.

2008 The Fortress of the Raven: Karak in the Middle Islamic Period (1100-1650) (Islamic History and Civilization, Studies and Texts 72), Leiden - New York - Köln 2008.

NuCCIOTTI, $\mathrm{M}$.

2007 Analisi stratigrafiche degli elevati: primi risultati: G. VANNINI (ed.), Archeologia dell'insediamento crociato-ayyubide in Transgiordania. Il progetto Shawbak, Firenze 2007, pp. 27-56.

in stampa Il palazzo Ayyubide di Shawbak: analisi archeologica e contestualizzazione storica: S. LUSUARDI SIENA (ed.), in stampa (www.academia.edu).

PAUTY, E.

1931 Catalogue Général du Musée Arabe: Les bois sculptés jusqu'à l'époque ayyoubide, Le Caire 1931. 
PRINGLE, D.

1997 Secular Buildings in the Crusader Kingdom of Jerusalem. An Archaeological Gazetteer, RABBAT, N.O

Cambridge 1997.

1995a The Citadel of Cairo. A New Interpretation of Royal Mamluk Architecture (Islamic History and Civilization, Studies and Texts 14), Leiden - New York - Köln 1995.

1995b The Ideological Significance of the Dār al-'Adl in the Medieval Islamic Orient: Journal of Middle Eastern Studies 27 (1995), pp. 3-28.

Rousset, M.-O.

1996 Evolution of a settlement in Mayādīn (Syria): K. BARTL - S. HAUSER (eds.), Continuity and Change in Northern Mesopotamia from the Hellenistic to the Early Islamic Period (Berliner Beiträge zum Vorderem Orient 17), Berlin 1996, pp. 185-194.

RUGIADI, M.

2007 Architectural Survey: The Ayyubid palace (CF34): G. VANNinI - M. NucciotTi (eds.), 'Medieval' Petra - Shawbak Project. Archaeological Season 2007. Field Report 2007, Amman 2007, relazione consegnata a Department of Antiquities of Jordan (www.shawbak.net), pp. 70-73.

SAUVAGET, J.

1928 Deux sanctuaires chiites d'Alep (second article): Syria IX (1928), pp. 320-327.

SAuvaget, J. - ÉCOChard, M. - SOURDEL-Thomine, J.

1938-1950 Les monuments ayyoubides de Damas, 4 livraisons (Institut français du Proche-Orient), Paris 1938-1950.

SAYYID, H.I.

1987 The Development of the Cairene Qā‘a: Some Considerations: Annales Islamologiques 23 (1987), pp. 31-53.

SOURDEL, D.

$1974 \quad$ Kal'at Nadjm: Encyclopaedia of Islam ${ }^{2}$ 1974, p. 482.

TABBAA, Y.

1997 Constructions of Power and Piety in Medieval Aleppo, Pennsylvania 1997.

2006 Defending Ayyubid Aleppo: the Fortifications of al-Ẓāhir Ghāzī (1186-1216): H. KenNEDy (ed.), Muslim Military Architecture in Greater Syria. From the Coming of Islam to the Ottoman Period, Leiden 2006, pp. 176-182.

TOUEIR, K

1985 Der Qașr al-Banāt in ar-Raqqa: Ausgrabung, Rekonstruktion und Wiederaufbau (19771982): Damaszener Mitteilungen 2 (1985), pp. 297-319.

VANNINI, G. (ed.)

2007 Archeologia dell'insediamento crociato-ayyubide in Transgiordania. Il progetto Shawbak, Firenze 2007.

VANNINI, G. - NuCCIOTTI, M. (eds.)

2007 'Medieval' Petra - Shawbak Project. Archaeological Season 2007. Field Report 2007, Amman 2007, relazione consegnata a Department of Antiquities of Jordan (www.shawbak.net).

2009 Da Petra a Shawbak, Archeologia di una frontiera, Firenze 2009.

Yovitchitch, C.

2006 The tower of Aybak in 'Ajlūn castel: an example of the spread of an architectural concept in early $13^{\text {th }}$-century Ayyubid fortification: H. KENNEDY (ed.), Muslim Military Architecture in Greater Syria. From the Coming of Islam to the Ottoman Period, Leiden 2006, pp. 225-243. 


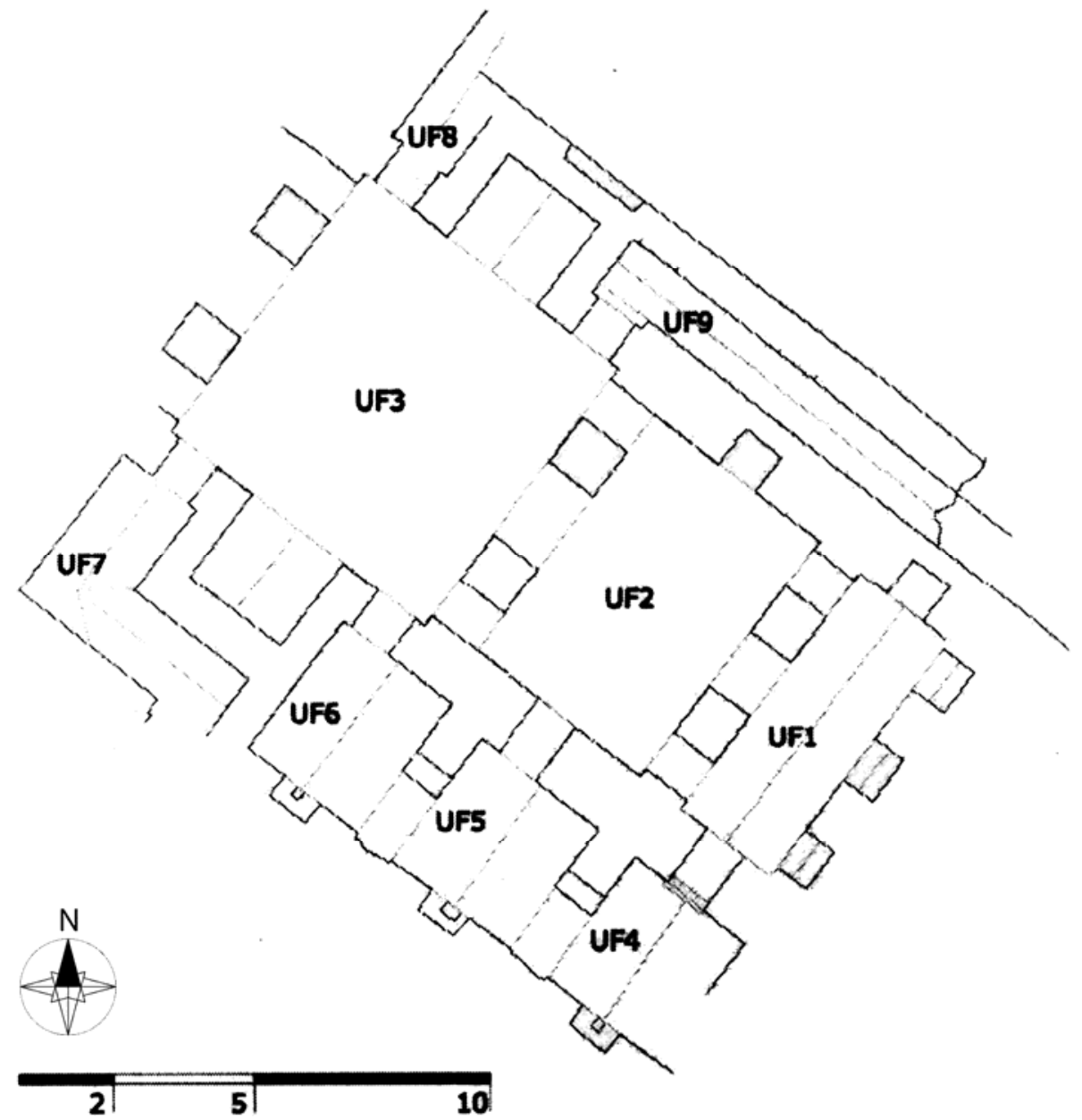

Fig. 1: Pianta del complesso di ricevimento del palazzo ayyubide (Progetto Shawbak, elaborazione Missione archeologica Università di Firenze). 


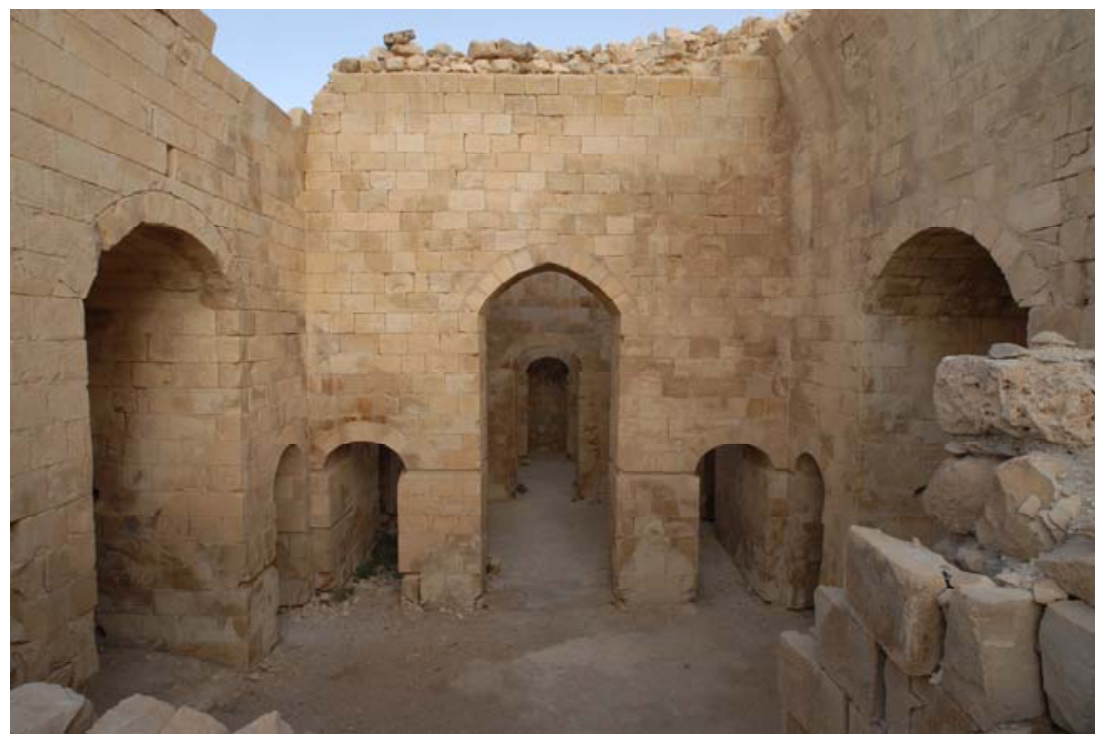

Fig. 2: La sala principale UF3, con i due īwān. Al centro, la cortina tripartita verso l'ambiente UF2, visibile attraverso l'arco centrale (Progetto Shawbak, foto Missione archeologica Università di Firenze).

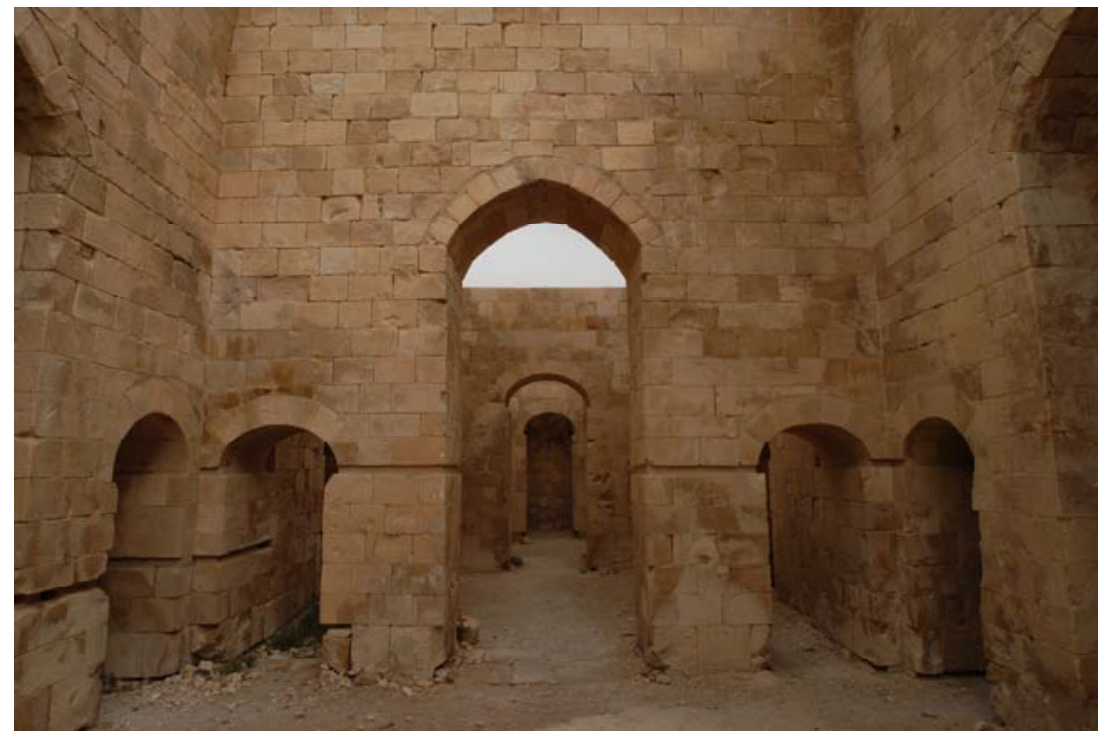

Fig. 3: UF3, la cortina tripartita verso UF2. Attraverso l'arco centrale sono visibili quello in UF2 e la nicchia di fondo nell'ambiente UF1 (Progetto Shawbak, foto Missione archeologica Università di Firenze). 


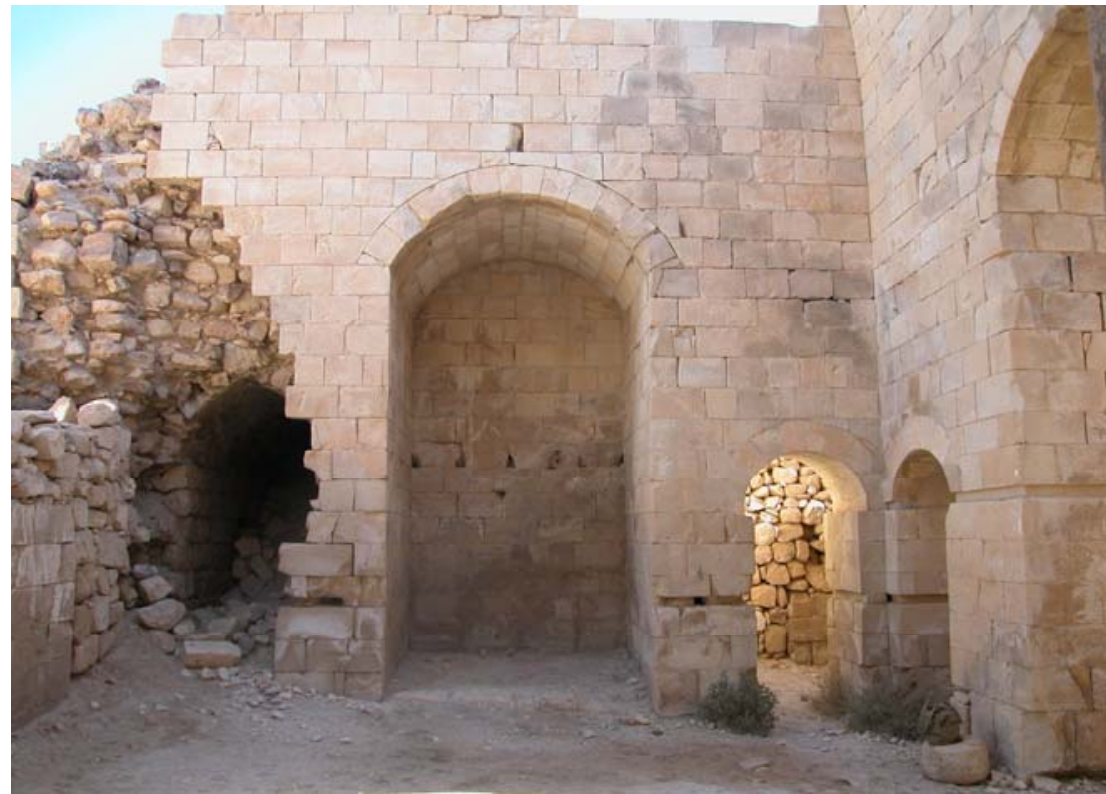

Fig. 4: UF3, il lato nord. Al centro l'īwān, ai lati i passaggi ad arco verso i corridoi UF8 e UF9 (Progetto Shawbak, foto Rugiadi 2007).

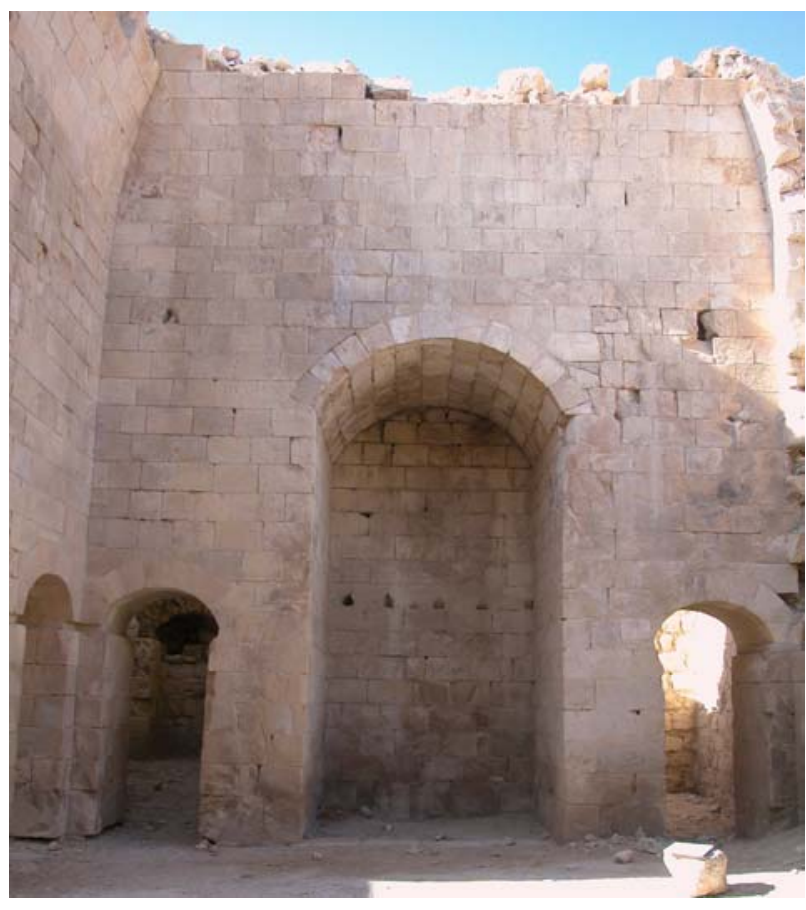

Fig. 5: UF3, il lato sud. Al centro l'īwān, ai lati i passaggi ad arco verso l'ambiente UF6 e il corridoio UF7 (Progetto Shawbak, foto Rugiadi 2007). 


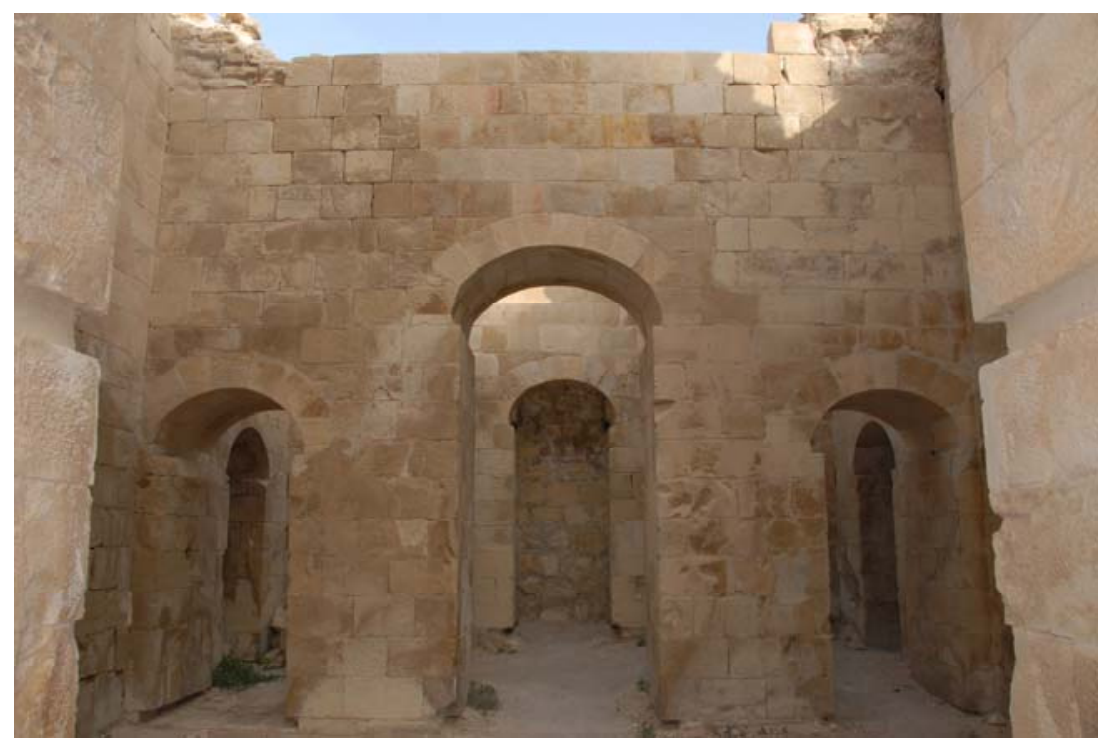

Fig. 6: UF2, la cortina tripartita est verso UF1. Attraverso l'arco centrale è visibile la nicchia di fondo in UF1; attraverso gli archi laterali sono visibili rispettivamente, sempre in UF1, la nicchia nord e l'arco sud (Progetto Shawbak, foto Missione archeologica Università di Firenze).

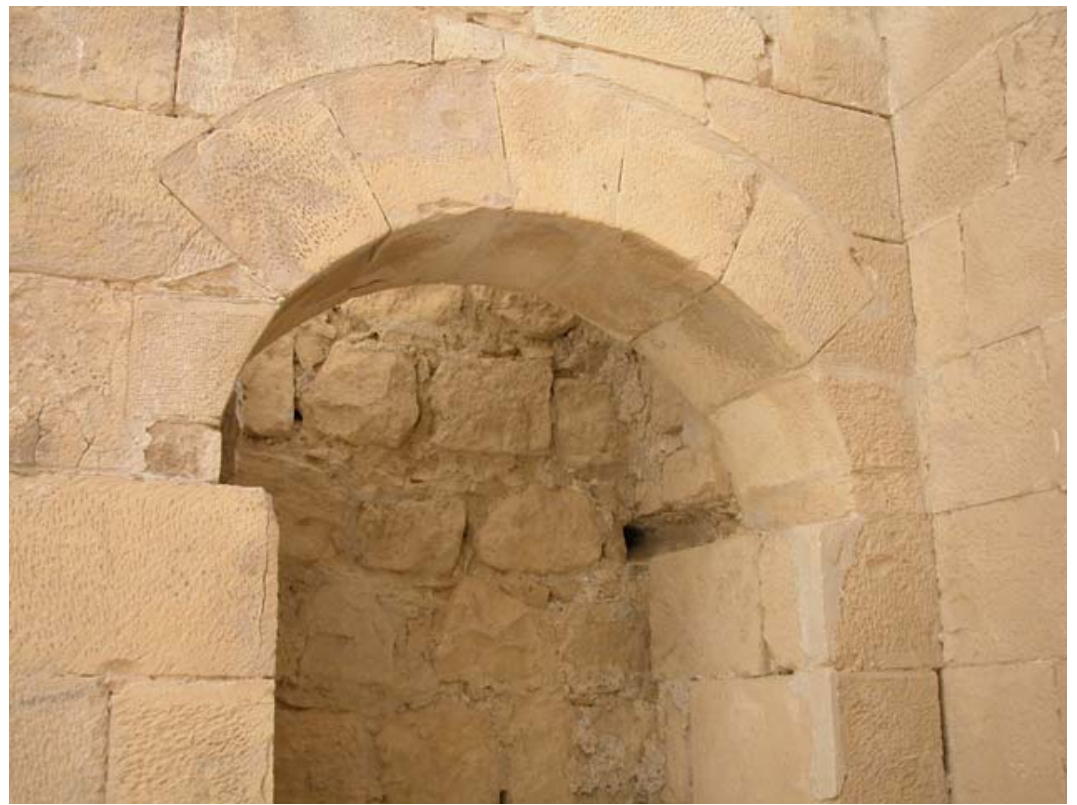

Fig. 7: UF1, una delle nicchie (Progetto Shawbak, foto Rugiadi 2007). 